\title{
Sensitivity analysis on a three-phase plant-wide water and resource recovery facility model for identification of significant parameters
}

\section{DS Ikumi ${ }^{1}$}

${ }^{1}$ Future Water Institute, New Engineering Building (NEB), University of Cape Town, Rondebosch, 7701, Cape Town, South Africa

Water and resource recovery facility (WRRF) mathematical models have been advancing towards their widespread application for sizing and operation of treatment plants to minimize energy consumption and cost while maximizing nutrient recovery and effluent quality. Effective utilisation of these models requires that they are well calibrated. However, difficulties (with important parameters not identified and uncertainties in interpretation of model output results) can be experienced in model calibration, especially due to (i) the intricate relationships of model output variables with model input factors (where parameters are inter-related to various model outputs), resulting in non-linearity, and (ii) the limitations (due to expensive and/or time-consuming experimental methods) experienced in procuring and reconciling data required for determination of the model input factors. This paper presents the performance of a sensitivity analysis, reinforced with expert-based reasoning, on a three-phase (aqueous-gas-solid) plant-wide model (PWM_SA, Ikumi et al., 2015), for identification of significant parameters, and highlights the ones requiring experimental determination, specific to the system. The sensitivity analysis exercise was performed using two methods i.e., Morris screening (screening method) and standardised regression coefficient (SRC; based on regression). This process was useful towards detection of the parameters, which are not normally measured at WRRFs, but may require attention for future application of mathematical models in decision-making processes for WRRFs. These included the influent fractions of unbiodegradable and readily biodegradable organics, the kinetic constants for hydrolysis of biodegradable particulates, the elemental composition of the organics and the specific growth rate of autotrophic nitrifying biomass.

\section{INTRODUCTION}

Continuous advancements are being made towards a more system-wide approach to modelling waste treatment systems, that incorporate the fate of the products being generated (e.g., mineral precipitates, stable organic sludge, biogas, etc.) in view of resource recovery. Because the functions of these water and resource recovery facilities (WRRFs) stretches beyond simply meeting effluent requirements (i.e., also includes optimisation of products to be generated), a high level of accuracy in predicting system response is required. However, with the increased size of these mathematical models, difficulties (with important model parameters not identified and uncertainties in intepretation of model output results) can be experienced in model calibration, especially due to the non-linearity brought about by (i) the intricate relationships of model output variables with model input factors (where parameters are inter-related to various model outputs) and (ii) the limitations experienced in procuring and reconciling data (due to expensive and/or time-consuming experimental methods) required for determination of the model input factors (this is especially when the model has significantly large numbers of unknown parameters and model components). Further, the inclusion of phosphorus (P) into system-wide models that could mimic the continuously evolving WRRFs has resulted in various complexities that necessitate a rigourous and systematic method of determining significant parameters and their values for confidence in the model predicted outputs. Phosphorus is removed from wastewater by transforming it from the dissolved liquid phase to the intracellular solid phase. Hence, for system-wide models, it was noted that the anaerobic digestion (AD) of P-rich sludge from biological excess P removal (EBPR) activated sludge (AS) systems, requires three-phase mixed weak acid/base chemistry because the release of biomass $\mathrm{P}$ or polyphosphate (PP) not only affects the system alkalinity but also can induce mineral (e.g., struvite) precipitation (Van Rensburg et al., 2003; Harding et al., 2010).

Various research groups have worked both collaboratively, and separately on related topics, towards development of WRRF mathematical models that integrate bioprocess stoichiometry and physicochemical transformations, for inclusion of processes such as nutrient release and multiple mineral precipitation (Batstone et al., 2012; Kazadi et al., 2015; Flores Alsina et al., 2016; Wang et al., 2016). Bioprocess stoichiometry and physicochemical transformations are also included in the presentation of a new 'three-phase' (aqueous-gas-solid) plant-wide model that includes P, (PWM_SA) (Brouckaert et al., 2010; Ikumi et al., 2015) which includes compatible activated sludge (AS; ASM2-3P) and anaerobic digestion (AD; UCTSDM3P) bioprocess model components and uses strict massbalance principles to track $P$ through the unit processes of a WRRF (with recognition of its impact on the mutual interaction between the connected unit operations). This paper presents the performance of a sensitivity analysis on a three-phase (aqueous-gas-solid) plant-wide model (PWM_SA, Ikumi et al., 2015), for identification of significant parameters and highlights the ones requiring experimental determination, specific to the system (i.e., where a generic value from literature cannot be used).
CORRESPONDENCE

DS Ikumi

EMAIL

david.ikumi@uct.ac.za

\section{DATES}

Received: 16 July 2019

Accepted: 23 June 2020

\section{KEYWORDS}

sensitivity analysis activated sludge anaerobic digestion plant-wide modelling

\section{COPYRIGHT}

(c) The Author(s)

Published under a Creative Commons Attribution 4.0 International Licence (CC BY 4.0) 
Sensitivity analysis has been applied in various studies as part of the standard processes of calibration of water and wastewater treatment system models (Vanrolleghem et al., 2003; Brun et al., 2001; Ikumi et al., 2014). Different methods have been applied based on the objectives of the study and the complexity of the model involved. Some applications in the field of anaerobic digestion (AD) modelling include: (i) the use of non-dimensional logarithmic sensitivity functions (i.e., partial derivatives of the state variables) by Noykova and Gyllenberg (2000) to compare the influence of different parameters and variables in a modified version of the Hill and Bath (1977) AD model; (ii) the application of decoupled direct method (DDM; i.e., decoupling the auxiliary equations from the model equations) applied by Silva and De Bortoli (2020) for an AD model of cellulose degradation for biogas production; (iii) calculation of the sensitivity index (Sobol, 1993) to define the most sensitive parameters for production of biogas (i.e., the methane yield), using the International Water Association (IWA) Anaerobic Digestion Model No. 1 (ADM1; Batstone et al., 2002) with optimized kinetic parameters in the anaerobic digestion of food waste (Zhao et al., 2019). As noted from the literature sources, various methods can be used in identification of influential model parameters. There have been some comparisons between various methods of sensitivity analysis (Neumann, 2012; Cosenza et al., 2013). Some of the methods that stood out in these comparisons included standard regression coefficient (SRC; is a linear correlation-based approach) method, Morris screening (Morris, 1991; determination of the elementary effects) and Extended-FAST (Fourier Amplitude Sensitivity Testing; an analysis of variance-based approach).

Sensitivity analysis objectives usually considered are factor prioritisation (identifying the model parameters with the greatest effect on model outputs) or factor fixing (identifying noninfluential factors that could be 'fixed') (Neumann, 2012; Mannina et al., 2011). However, modellers may find it useful to identify both important (factor prioritization) and non-influential (factor fixing) input parameters. In the study conducted by Neumann (2012) it was shown that, although the SRC method was applied outside its' validity range, it still identified similar important parameters to Extended-FAST. In applying a sensitivity analysis of the UCTSDM3P model of Ikumi et al. (2015) used to simulate an upflow anaerobic sludge blanket (UASB) reactor, Ghoor (2020), noted the SRC method to be useful in factor prioritisation, although it assumes existence of a linear relationship between input parameters and output variables. Ghoor (2020) notes that, despite this linear relationship not being true for bioprocess models such as that for $\mathrm{AD}$ systems, the correlation coefficient of greater than 0.7 can allow for an assumption that the applied linear model in SRC explains the relationships reasonably well and accounts for $70 \%$ of the variance in the data. This made the SRC simpler to understand, when compared with the more complex methods such as ExtendedFAST. For similar reasons to those stated by Ghoor (2020), the SRC method was selected to be used in factor prioritisation for this study. However, because it was deemed useful to assess both linear and/or non-linear effects of all the model parameters on the output variables (Mannina et al., 2011), Morris screening was selected as a second method of analysis because of its capabilities in factor fixing and identifying non-linear relations between parameters and variables (Gamerith et al., 2011).) Further, Morris screening was recommended by Herman et al. (2013) as an efficient method that can identify the most and least sensitive parameters, similar to a more complex variance-based Sobol sensitivity index method (Sobol, 1993), at a reasonable computational cost.

\section{EXPERIMENTAL SYSTEM LAYOUT}

The experimental layout of Ikumi (2011) is used in this study. It replicates at laboratory scale three WWTP schemes, comprising (i) a Modified Ludzack-Ettinger (MLE) nitrification-denitrification (ND) activated sludge (AS) system treating raw sewage (MLE 1) with anaerobic digestion (AD) of its waste activated sludge (WAS) in AD system number 1 (i.e., AD1), (ii) an identical MLE system (MLE 2) treating settled sewage with AD of its WAS in $\mathrm{AD} 2$, and (iii) a membrane (MBR) University of Cape Town (UCT) ND enhanced biological P removal (NDEBPR) system treating settled sewage with (i) AD of its WAS in AD3. All three AS systems (UCT, MLE 1 and MLE 2) were operated at steady state, at a 10-day system sludge age (SRT), and were fed the same $600 \mathrm{mgCOD} / \mathrm{L}$ settled wastewater, except that the UCT system influent included same additives (i.e., $200 \mathrm{mgCOD} / \mathrm{L}$ acetate and $40 \mathrm{mg} / \mathrm{L} \mathrm{P}$ from di-potassium hydrogen phosphate $\left(\mathrm{KH}_{2} \mathrm{PO}_{4}\right)$ ). The MLE systems had no chemical additives but to one of them (MLE 2) a measured constant flux (gCOD/d) of macerated PS collected from the Athlone WWTP (Cape Town) was added to the same settled WW to make up raw sewage influent and increase its COD from the basic $600 \mathrm{mgCOD} / \mathrm{L}$ to $1000 \mathrm{mgCOD} / \mathrm{L}$. The added PS, the WAS from the three AS systems, and a blend of PS - MLE1 WAS were also anaerobically digested. Hence, the AD systems constituted 5 separate flow-through anaerobic digestion (AD) systems operated successively at different solid retention times (SRTs). To initiate the calibration process, the sensitivity analysis procedure was perfomed with simulation of the MBR UCT NDEBPR system and the AD system that digested its WAS (Fig. 1 shows the experimental set-up). Table 1 and Table 2 show the operating parameters for the UCT AS and AD systems, respectively. The prepared experimental set-up allowed for the tracking of COD, $\mathrm{N}$ and $\mathrm{P}$ through the aerobic and anaerobic unit processes of the WWTP. Table 3 presents a guide indicating all measurements performed on samples taken from the unit processes of the plant configuration.

Table 1. Design and operating parameters for UCT AS system

\begin{tabular}{lc}
\hline Parameter & Value \\
\hline Sludge age (d) & 10 \\
Influent COD (mg/L) & $600+200^{\mathrm{a}}$ \\
Influent flow (L/d) & 150 \\
Waste flow (L/d) (from aerobic reactor) & 5.74 \\
Volume (L)/mass fractions: anaerobic & $19 ; 0.133$ \\
Volume (L)/mass fractions: anoxic & $21 / 0.275$ \\
Volume (L)/mass fractions: aerobic & $35 / 0.592$ \\
Recycle ratios: a (aerobic to anoxic) & $2.8-3.4^{\mathrm{b}}$ \\
Recycle ratios: $s$ (from settling tank) & --- \\
Recycle ratios: $r$ (anoxic to anaerobic) & $1.1-1.2^{\mathrm{b}}$ \\
HRT - nominal/actual (h): anaerobic & $3.04 / 1.41$ \\
HRT - nominal/actual (h): anoxic & $3.36 / 0.61$ \\
HRT - nominal/actual (h): aerobic & $5.6 / 1.27$ \\
\hline
\end{tabular}

${ }^{a}$ Dosed $200 \mathrm{mgCOD} / \mathrm{L}$ sodium acetate; ${ }^{b}$ the MBR UCT a recycle varied from 2.8 to 3.4 and s recycle varied from 1.1 to 1.2 during its operation. 
Table 2. Design and operating parameters for AD system

\begin{tabular}{|c|c|c|c|c|c|}
\hline Test period & 1 & 2 & 3 & 4 & 5 \\
\hline Period dates & 1 Feb-08 Apr & 9 Apr-04 Jul & 1 Feb-12-Jun & $5 \mathrm{Jul}-28 \mathrm{Aug}$ & 28 Aug-2 Nov \\
\hline Period duration & 68 & 87 & 133 & 55 & 66 \\
\hline WW batches used & $13-14$ & $15-16$ & $16-17$ & $18-19$ & $20-21$ \\
\hline AD sludge age & $18 \mathrm{~d}$ & $40 \mathrm{~d}$ & $60 d$ & $25 d$ & $10 \mathrm{~d}$ \\
\hline Flow (L/d) & 0.89 & 0.4 & 0.08 & 0.64 & 1.6 \\
\hline Flux (gCOD/d) & 8 & 3.6 & 0.72 & 5.76 & 14.4 \\
\hline
\end{tabular}

$A D$ volume was always $16 L$ apart from $60 d A D$ which was operated using small $5 L A D$ volume reactors, The NDEBPR WAS taken from AS system was $5.7 \mathrm{~L} / \mathrm{d}$ at a concentration of about $9 \mathrm{gCOD} / \mathrm{L}$. The required volume of this WAS was fed to the AD without thickening.

Table 3. Sampling points and parameter measurement

\begin{tabular}{|c|c|c|c|c|c|c|c|c|c|c|c|c|c|c|c|c|}
\hline Test & COD & TKN & VFA & FSA & $\mathrm{NO}_{3}$ & $\mathrm{NO}_{2}$ & TP & OP & $\mathrm{Me}^{+}$ & Alk & TSS & VSS & OUR & DSVI & $\mathrm{pH}$ & Gas (vol\& \% CO2) \\
\hline Influent & F; UF & UF & UF & $\mathrm{F}$ & & & UF; F & $\mathrm{F}$ & UF; F & UF & & & & & & \\
\hline Anaerobic & & & & & $F$ & $F$ & $\mathrm{~F}$ & & & & UF & UF & & & & \\
\hline Anoxic & & & & & $\mathrm{F}$ & $\mathrm{F}$ & $\mathrm{F}$ & & & & UF & UF & & & & \\
\hline Aerobic & UF & UF & & & $\mathrm{F}$ & $\mathrm{F}$ & UF & & UF; F & & UF & UF & $\mathrm{D}$ & $\mathrm{D}$ & $\mathrm{D}$ & \\
\hline Final effluent & $\mathrm{F}$ & $F ;$ UF & $\mathrm{F}$ & $F$ & $\mathrm{~F}$ & $\mathrm{~F}$ & F; UF & $\mathrm{F}$ & & $\mathrm{F}$ & & & & & & \\
\hline$A D$ influent & F; UF & F; UF & UF & $\mathrm{F}$ & & & F; UF & $\mathrm{F}$ & F; UF & UF & UF & UF & & & & \\
\hline AD effluent & F; UF & F; UF & $\mathrm{F}$ & $\mathrm{F}$ & & & F; UF & $\mathrm{F}$ & $\mathrm{F}$ & $\mathrm{F}$ & UF & UF & & & $\mathrm{D}$ & $\mathrm{D}$ \\
\hline
\end{tabular}

$F=0.45 \mu m$ filtered; UF = unfiltered samples; $D=$ direct measurement taken.

COD (chemical oxygen demand), TKN (total Kjeldahl nitrogen), FSA (free and saline ammonia), TP (total phosphorus), OP (ortho-P), TSS (total suspended solids), VSS (volatile suspended solids) according to Standard Methods (1998). $\mathrm{NO}_{3}$ (nitrate) and $\mathrm{NO}_{2}$ (nitrite) by Technicon Autoanalyzer Industrial Method 33.68 and 35.67W; Me+ (metals - Mg, K, Ca) by acid digestion of unfiltered (UF) and filtered (F) samples followed by atomic adsorption (AA) analysis. DSVI (diluted sludge volume index) according to Ekama and Marais (1984); OUR (oxygen utilization rate) measured directly in aerobic reactor according to Randall et al. (1991). VFA (volatile fatty acids) and $\mathrm{H}_{2} \mathrm{CO}_{3}$ alkalinity with the 5-point titration of Moosbrugger et al. (1992); gas volume was measured by gas volume counter directly connected to $A D$; gas was collected in $5 \mathrm{~L}$ Tedlar gas bags and $\mathrm{CO}_{2}$ and $\mathrm{CH}_{4}$ composition analysed by gas chromatograph.

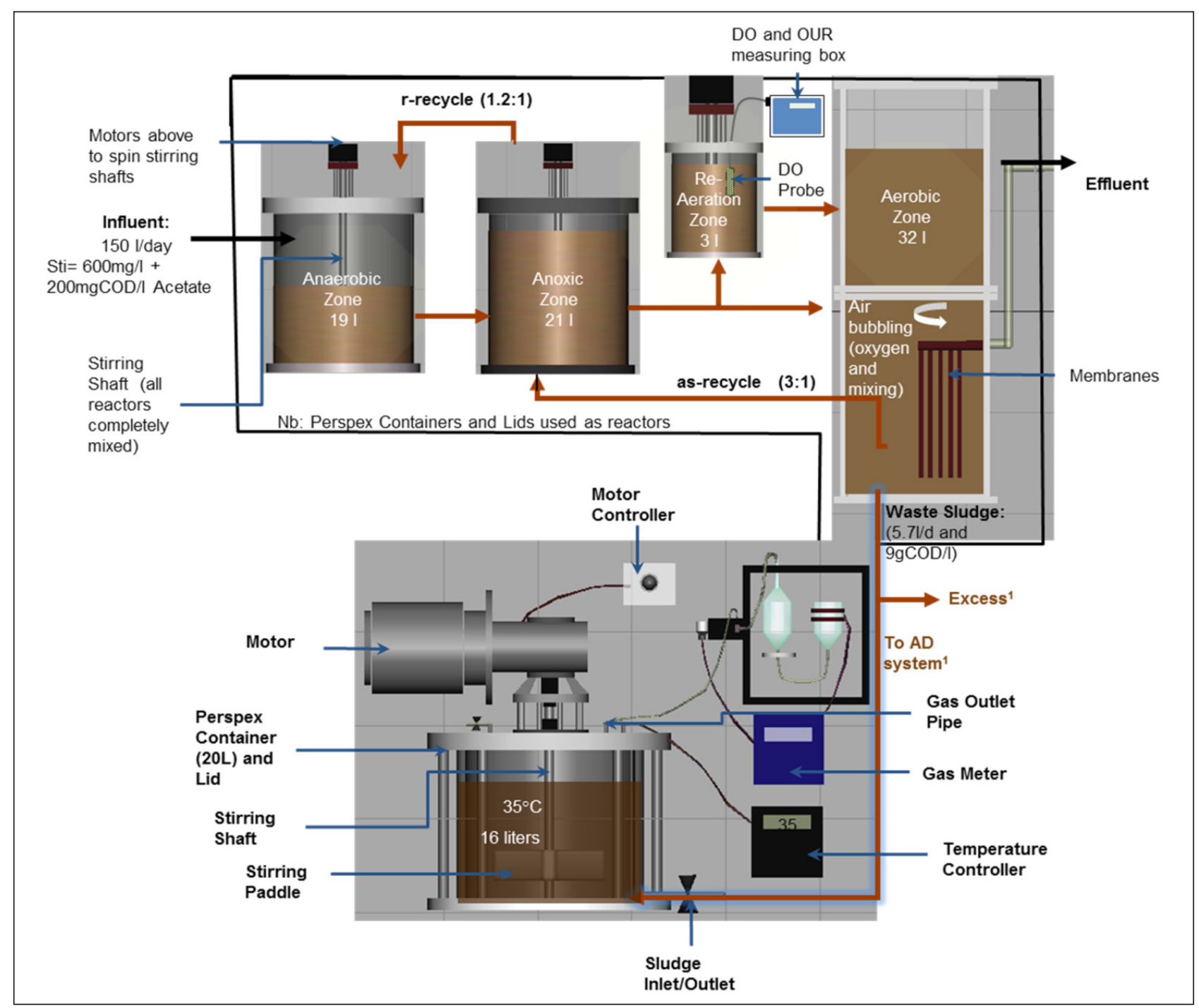

Figure 1. Simulated experimental set-up used to carry out research investigation 


\section{MODEL DESCRIPTION AND VERIFICATION}

The UCT three-phase plant-wide model (Ikumi et al., 2015) was developed for simulating the biological processes to track and predict the output of materials $(\mathrm{COD}$, carbon $(\mathrm{C})$, hydrogen $(\mathrm{H})$, oxygen $(\mathrm{O})$, nitrogen $(\mathrm{N})$, phosphorus $(\mathrm{P})$, magnesium $(\mathrm{Mg})$, potassium $(\mathrm{K})$ and calcium $(\mathrm{Ca})$ ) along the unit processes of a WWTP. It comprises three sub-models, integrated for simulation of the entire WWTP under various configurations (e.g., NDBEPR AS system linked to an $\mathrm{AD}$ or an anoxic-aerobic digestion (AAD) for WAS stabilisation). These sub-models include:

1. The ionic speciation model (Brouckaert et al., 2010). This model includes pairing of ionic components (the set of model ionic species is given in Table 4) and inter-phase transfers of component species. Table 5 gives an example of a set of equilibrium and mass-balance equations used in the ionic speciation subroutine.

2. The ASM2-3P model: This is the Activated Sludge Model No. 2 (ASM2, Henze et al., 1995), modified to include the ionic speciation model (Brouckaert et al., 2010), the Inorganic Settleable Solids (ISS) model of Ekama and Wentzel (2004) and including multiple mineral precipitation according to Musvoto et al. (2000a,b).

3. The UCTSDM3P Model: This is the University of Cape Town Anaerobic Digestion Model (UCTADM; Sötemann et al., 2005), modified to include the hydrolysis of multiple organic sludge types (PS, ND WAS, NDBEPR WAS and PS-WAS blends), the Ekama and Wentzel (2004) ISS model, multiple mineral precipitation processes according to Musvoto et al. (2000a, b) and the Brouckaert et al. (2010) aqueous speciation model which facilitates ionic speciation (Ikumi et al., 2015).

For their compatibility, the ASM2-3P and UCTSDM3P models have the same comprehensive set of model components (supermodel approach, Volcke et al., 2006; model components given in Table 6 and applied stoichiometric processes in Table 7), including parameterized stoichiometry for the bioprocesses and sharing the same ionic speciation subroutine model. All the model components and parameters were defined and named according to the standard notational framework proposed by Corominas et al. (2010).

Model verification: To initiate the evaluation of the PWM_SA model, the systematic method proposed by Hauduc et al. (2010) was applied to verify that material (COD, C, $\mathrm{H}, \mathrm{O} \mathrm{N}, \mathrm{P}, \mathrm{Mg} \mathrm{K}$ and $\mathrm{Ca}$ ) balances were achieved in the determination of all stoichiometric processes.

Parameter values: The initial (prior) values for suitable kinetic and stoichiometric parameters as obtained experimentally or from literature were entered, and given the typical value range ( $\theta \mathrm{i}$.min to $\left.\theta i \_m a x\right)$, determined according to the methods proposed by Brun et al. (2002) considering 3 classes:

- Accurately known - these have a relative uncertainty of 5\% (Class 1)

- Moderately inaccurately known parameters with a relative uncertainty of $20 \%$ (Class 2)

- Very poorly known parameters with a relative uncertainty of $50 \%$ (Class 3)

Tables 8 and 9 show the model parameters for ASM2-3P and UCTSDM3P, respectively.

Table 4. Ionic species selected for the three-phase model (PWM_SA)

\begin{tabular}{|c|c|c|c|c|c|}
\hline & Formula & Description & & Formula & Description \\
\hline 1 & $\mathrm{H}^{+}$ & Hydrogen ion & 23 & $\mathrm{NH}_{4} \mathrm{SO}_{4}^{-}$ & Ammonium sulphate \\
\hline 2 & $\mathrm{Na}^{+}$ & Sodium & 24 & $\mathrm{MgPO}_{4}^{-}$ & Magnesium phosphate \\
\hline 3 & $\mathrm{~K}^{+}$ & Potassium & 25 & $\mathrm{CaCH}_{3} \mathrm{COO}^{+}$ & Calcium acetate \\
\hline 4 & $\mathrm{Ca}^{2+}$ & Calcium & 26 & $\mathrm{CaCH}_{3} \mathrm{CH}_{2} \mathrm{COO}^{+}$ & Calcium propionate \\
\hline 5 & $\mathrm{Mg}^{2+}$ & Magnesium & 27 & $\mathrm{CaHCO}_{3}^{+}$ & Calcium bicarbonate \\
\hline 6 & $\mathrm{NH}_{4}^{+}$ & Ammonium & 28 & $\mathrm{NaSO}_{4}^{-}$ & Sodium sulphate \\
\hline 7 & $\mathrm{Cl}^{-}$ & Chloride & 29 & $\mathrm{MgHPO}_{4}$ & Magnesium hydrogen phosphate \\
\hline 8 & $\mathrm{CH}_{3} \mathrm{COO}^{-}$ & Acetate & 30 & $\mathrm{CH}_{3} \mathrm{COONa}$ & Sodium acetate \\
\hline 9 & $\mathrm{CH}_{3} \mathrm{CH}_{2} \mathrm{COO}^{-}$ & Propionate & 31 & $\mathrm{H}_{2} \mathrm{CO}_{3}$ & Di-hydrogen carbonate \\
\hline 10 & $\mathrm{CO}_{3}^{2-}$ & Carbonate & 32 & $\mathrm{MgSO}_{4}$ & Magnesium sulphate \\
\hline 11 & $\mathrm{SO}_{4}^{2-}$ & Sulphate & 33 & $\mathrm{HPO}_{4}^{2-}$ & Hydrogen phosphate \\
\hline 12 & $\mathrm{PO}_{4}^{3-}$ & Phosphate & 34 & $\mathrm{NH}_{3}$ & Ammonia \\
\hline 13 & $\mathrm{NO}_{3}^{-}$ & Nitrate & 35 & $\mathrm{MgCO}_{3}$ & Magnesium carbonate \\
\hline 14 & $\mathrm{OH}^{-}$ & Hydroxide ion & 36 & $\mathrm{ACPO}_{4}^{-}$ & Calcium phosphate \\
\hline 15 & $\mathrm{CH}_{3} \mathrm{COOH}$ & Acetic acid & 37 & $\mathrm{MgHCO}_{3}^{+}$ & Magnesium hydrogen carbonate \\
\hline 16 & $\mathrm{CH}_{3} \mathrm{CH}_{2} \mathrm{COOH}$ & Propionic acid & 38 & $\mathrm{CaHPO}_{4}^{-}$ & Calcium hydrogen phosphate \\
\hline 17 & $\mathrm{HCO}_{3}^{-}$ & Bi carbonate & 39 & $\mathrm{NaCO}_{3}^{-}$ & Sodium carbonate \\
\hline 18 & $\mathrm{CaSO}_{4}$ & Calcium sulphate & 40 & $\mathrm{MgH}_{2} \mathrm{PO}_{4}^{+}$ & Magnesium di-hydrogen phosphate \\
\hline 19 & $\mathrm{H}_{2} \mathrm{PO}_{4}^{-}$ & Di-hydrogen phosphate & 41 & $\mathrm{NaHCO}_{3}$ & Sodium hydrogen carbonate \\
\hline 20 & $\mathrm{MgCH}_{3} \mathrm{COO}^{+}$ & Magnesium acetate & 42 & $\mathrm{NaHPO}_{4}^{-}$ & Sodium hydrogen phosphate \\
\hline 21 & $\mathrm{MgCH}_{3} \mathrm{CH}_{2} \mathrm{COO}^{+}$ & Magnesium propionate & 43 & $\mathrm{CaOH}^{+}$ & Calcium hydroxide \\
\hline 22 & $\mathrm{CaCO}_{3}$ & Calcium carbonate & 44 & $\mathrm{MgOH}^{+}$ & Magnesium hydroxide \\
\hline
\end{tabular}

Table 5. Example for equilibrium and mass balance equations for ionic speciation

\begin{tabular}{ll}
\hline Weak acid sub-system & *Aqueous phase equilibrium equations \\
\hline & {$\left[\mathrm{NH}_{3}\right]=\frac{\left.\mathrm{K}_{\mathrm{NH}_{4} \cdot[\mathrm{NH}+}^{+}\right]}{\left(\mathrm{H}^{+}\right)}$} \\
& {$\left[\mathrm{NH}_{4} \mathrm{SO}_{4}^{-}\right]=\frac{\left[\mathrm{SO}_{4}^{2-}\right]\left[\mathrm{NH}_{4}^{+}\right]}{\mathrm{K}_{\mathrm{NH}_{4} \mathrm{SO}_{4}}}$} \\
Ammonia & {$\left[\mathrm{NH}_{x}\right]=\left[\mathrm{NH}_{4}^{+}\right]+\left[\mathrm{NH}_{3}\right]+\left[\mathrm{NH}_{4} \mathrm{SO}_{4}^{-}\right]$} \\
\hline
\end{tabular}

*Where $\left(H^{+}\right)$is the hydrogen ion activity, $[X]$ the molar concentrations of species $X$ and $K_{X^{\prime}}$ is the thermodynamic equilibrium constant for species $X$, adjusted for Debye Hückel effects to account for the activity of ions in low salinity water (Stumm and Morgan, 1996). 
Table 6. The universally selected model components for UCT three-phase plant-wide model (PWM_SA)

\begin{tabular}{|c|c|c|}
\hline Component description & Empirical formula & Name/notation \\
\hline Water & $\mathrm{H}_{2} \mathrm{O}$ & $\mathrm{H}_{2} \mathrm{O}$ \\
\hline Hydrogen ion & $\mathrm{H}^{+}$ & S_H \\
\hline Sodium & $\mathrm{Na}^{+}$ & S_Na \\
\hline Potassium & $\mathrm{K}^{+}$ & S_K \\
\hline Calcium & $\mathrm{Ca}^{2+}$ & S_Ca \\
\hline Magnesium & $\mathrm{Mg}^{2+}$ & S_Mg \\
\hline Ammonium & $\mathrm{NH}_{4}^{+}$ & S_NH $\mathrm{N}_{\mathrm{x}}$ \\
\hline Chloride & $\mathrm{Cl}^{-}$ & S_Cl \\
\hline Acetate & $\mathrm{CH}_{3} \mathrm{COO}^{-}$ & S_VFA \\
\hline Propionate & $\mathrm{CH}_{3} \mathrm{CH}_{2} \mathrm{COO}$ & S_Pr \\
\hline Carbonate & $\mathrm{CO}_{3}^{2-}$ & $\mathrm{S}_{-} \mathrm{CO}_{3}$ \\
\hline Sulphate & $\mathrm{SO}_{4}^{2-}$ & $\mathrm{S}_{-} \mathrm{SO}_{4}$ \\
\hline Phosphate & $\mathrm{PO}_{4}^{3-}$ & $\mathrm{S}_{-} \mathrm{PO}_{4}$ \\
\hline Nitrate & $\mathrm{NO}_{3}^{-}$ & $\mathrm{S} \_\mathrm{NO}_{\mathrm{x}}$ \\
\hline Dissolved hydrogen & $\mathrm{H}_{2}$ & $\mathrm{~S} \_\mathrm{H} 2$ \\
\hline Dissolved oxygen & $\mathrm{O}_{2}$ & S_O2 \\
\hline Unbiodegradable soluble organics & $\mathrm{CH}_{Y u} \mathrm{O}_{\mathrm{Zu}} \mathrm{N}_{\mathrm{Au}} \mathrm{P}_{\mathrm{Bu}}$ & $\bar{S} \_U$ \\
\hline Fermentable biodegradable soluble organics & $\mathrm{CH}_{\mathrm{Yf}} \mathrm{O}_{\mathrm{Zf}} \mathrm{N}_{\mathrm{Af}} \mathrm{P}_{\mathrm{Bf}}$ & S_F \\
\hline Glucose & $\mathrm{C}_{6} \mathrm{H}_{12} \mathrm{O}_{6}$ & S_Glu \\
\hline Unbiodegradable particulate organics & $\mathrm{CH}_{\text {Yup }} \mathrm{O}_{\text {Zup }} \mathrm{N}_{\text {Aup }} \mathrm{P}_{\text {Bup }}$ & X_U_inf \\
\hline Biodegradable particulate organics & $\mathrm{CH}_{\mathrm{Ybp}} \mathrm{O}_{z b \mathrm{p}} \mathrm{N}_{\mathrm{Abp}} \mathrm{P}_{\text {Bbp }}$ & X_B_Org \\
\hline Primary sludge biodegradable particulate organics & $\mathrm{CH}_{\text {Ybps }} \mathrm{O}_{\text {zbps }} \mathrm{N}_{\text {Abps }} \mathrm{P}_{\text {Bbps }}$ & X_B_Inf \\
\hline Polyphosphate & $\mathrm{K}_{\mathrm{kp}} \mathrm{Mg}_{\mathrm{mp}} \mathrm{Ca}_{\mathrm{cp}} \mathrm{PO}_{3}$ & X_PAO_PP \\
\hline Poly-hydroxy-alkanoate & $\mathrm{C}_{4} \mathrm{H}_{6} \mathrm{O}_{2}$ & X_PAO_Stor \\
\hline Struvite & $\mathrm{MgNH}_{4} \mathrm{PO}_{4} \cdot 6 \mathrm{H}_{2} \mathrm{O}$ & X_Str_NH4 \\
\hline Calcium phosphate & $\mathrm{Ca}_{3}\left(\mathrm{PO}_{4}\right)_{2}$ & $X \_A C P$ \\
\hline K-struvite & $\mathrm{MgKPO}_{4} \cdot 6 \mathrm{H}_{2} \mathrm{O}$ & X_Str_K \\
\hline Calcite & $\mathrm{CaCO} 3$ & $\bar{x}_{-} \mathrm{Cal}$ \\
\hline Magnesite & $\mathrm{MgCO} 3$ & X_Mag \\
\hline Newberyite & $\mathrm{MgHPO} 4$ & X_Newb \\
\hline Influent inorganic settleable solids & - & X_ISS \\
\hline Ordinary heterotrophic organisms & $\mathrm{CH}_{\mathrm{YO}_{0}} \mathrm{O}_{\mathrm{Zo}} \mathrm{N}_{\mathrm{Ao}} \mathrm{P}_{\mathrm{BO}}$ & $\mathrm{x} \_\mathrm{OHO}$ \\
\hline Phosphate accumulating organisms & $\mathrm{CH}_{\mathrm{YO}_{0}} \mathrm{O}_{\mathrm{ZO}} \mathrm{N}_{\mathrm{A0}} \mathrm{P}_{\mathrm{BO}}$ & X_PAO \\
\hline Autotrophic nitrifying organisms & $\mathrm{CH}_{\mathrm{Y}_{\mathrm{O}}} \mathrm{O}_{\mathrm{ZO}} \mathrm{N}_{\mathrm{A} 0} \mathrm{P}_{\mathrm{BO}}$ & X_ANO \\
\hline Acidogens & $\mathrm{CH}_{\mathrm{Yo}} \mathrm{O}_{\mathrm{Zo}} \mathrm{N}_{\mathrm{Ao}_{\mathrm{O}}} \mathrm{P}_{\mathrm{Bo}}$ & $X \_Z A D$ \\
\hline Acetogens & $\mathrm{CH}_{\mathrm{YO}} \mathrm{O}_{\mathrm{ZO}} \mathrm{N}_{\mathrm{Ao}} \mathrm{P}_{\mathrm{BO}}$ & X_ZAC \\
\hline Acetoclastic methanogens & 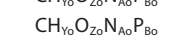 & X_ZAM \\
\hline Hydrogenotrophic methanogens & $\mathrm{CH}_{\mathrm{YO}_{0}} \mathrm{O}_{\mathrm{Z}_{0}} \mathrm{~N}_{\mathrm{AO}_{0}} \mathrm{P}_{\mathrm{BO}}$ & X_ZHM \\
\hline Endogenous residue & $\mathrm{CH}_{y e} \mathrm{O}_{z e} \mathrm{~N}_{\mathrm{ae}} \mathrm{P}_{\text {be }}$ & X_U_Org \\
\hline Carbon dioxide & $\mathrm{CO}_{2}$ & $\mathrm{G}_{-} \mathrm{CO}_{2}$ \\
\hline Methane & $\mathrm{CH}_{4}$ & $\mathrm{G}_{-} \mathrm{CH}_{4}$ \\
\hline
\end{tabular}

All components are presented in the units of $\mathrm{g} / \mathrm{m}^{3}$

Table 7. Processes used in the application of UCT three-phase plant-wide model

\begin{tabular}{|c|c|}
\hline Name & Description \\
\hline AerHydrol & Aerobic hydrolysis of biodegradable particulate organics (BPO) \\
\hline AnHydrol & Anoxic hydrolysis of BPO \\
\hline AnaerHydrol & Anaerobic hydrolysis of BPO \\
\hline AerGrowthOnSf & Aerobic OHO growth on fermentable soluble organics (FBSO) \\
\hline AerGrowthOnSa & Aerobic $\mathrm{OHO}$ growth on Acetate \\
\hline AnGrowthOnSfDenitrif & Anoxic $\mathrm{OHO}$ growth on FBSO \\
\hline AnGrowthOnSaDenitrif & Anoxic $\mathrm{OHO}$ growth on Acetate \\
\hline Fermentation & Fermentation of FBSO \\
\hline LysisOfAuto & Storage of poly-hydroxy-alkanoate (PHA) by PAOs \\
\hline StorageOfXPP & Aerobic storage of PP with PHA uptake \\
\hline AerGrowthOnXPHA & Aerobic growth of PAOs \\
\hline LysisOfXPP & Release and hydrolysis of polyphosphate (PP) \\
\hline LysisOfXPHA & Release and hydrolysis of PHA \\
\hline GrowthOfAuto & Aerobic growth of ANOs with nitrification \\
\hline OHO_Lysis & Lysis of OHOs in aerobic systems \\
\hline LysisOfXPAO & Lysis of PAOs in aerobic systems \\
\hline LysisOfAuto & Lysis of ANOs in AS system \\
\hline Aeration & Oxygen supply to aerobic reactor \\
\hline FSO_Hydrolysis & Hydrolysis of FBSO in AD system \\
\hline BPO_Hydrolysis & Hydrolysis of BPO produced by dead biomass \\
\hline BPO_PS_Hydrolysis & Hydrolysis of BPO from primary sludge (PS) \\
\hline OHO_Lysis_AD & Lysis of OHOs in AD system \\
\hline PAO_Lysis_AD & Lysis of PAOs in AD system \\
\hline PP_Release & Release of PP with uptake of PHA in AD system \\
\hline PP_Hydrolysis & Release and hydrolysis of PP in AD system \\
\hline PHA_Hydrolysis & Release and hydrolysis of PHA in AD system \\
\hline Acidogenesis_L & Low hydrogen partial pressure $\left(\mathrm{p}_{\mathrm{H} 2}\right)$ Acidogenesis \\
\hline Acidogenesis_H & High $\mathrm{p}_{\mathrm{H} 2}$ acidogenesis \\
\hline AD_Decay & Lysis of acidogens \\
\hline Acetogenesis & Growth of acetogens in AD system \\
\hline AC_Decay & Lysis of acetogens \\
\hline Acet_Methanogenesis & Growth of acetoclastic methanogens in AD system \\
\hline AM_Decay & Lysis of acetoclastic methanogens \\
\hline Hyd_Methanogenesis & Growth of hydrogenotrophic methanogens in AD system \\
\hline HM_Decay & Lysis of hydrogenotrophic methanogens \\
\hline
\end{tabular}


Table 8. Parameters used in simulating the ASM2-3P Model

\begin{tabular}{|c|c|c|c|c|c|c|}
\hline No. & Parameter & Description & Initial value & Units & Class & Uncertainty \\
\hline 1 & $\mathrm{KLA}_{\text {CO2 }}\left(\mathrm{K}_{\mathrm{lac}}\right)$ & $\mathrm{CO}_{2}$ liquid phase mass transfer rate coefficient & 1 & $1 / \mathrm{d}$ & 3 & 0.25 \\
\hline 2 & $\mathrm{~K}_{\text {__ } \_ \text {VFA }}\left(\mathrm{K}_{\text {Sac }}\right)$ & Saturation coeff. for S_A (acetate) & 4 & $\mathrm{gCOD} / \mathrm{m}^{3}$ & 3 & 2 \\
\hline 3 & $\mathrm{~K}_{\text {_S_ALK }}\left(\mathrm{K}_{\text {Salk }}\right)$ & Saturation coeff. for alkalinity $\left(\mathrm{HCO}_{3}^{-}\right)$ & 0.1 & $\mathrm{~mol} \mathrm{HCO} / \mathrm{m}^{3}$ & 3 & 0.05 \\
\hline 4 & $\mathrm{~K}_{\text {_S_ALK_ANO }}\left(\mathrm{K}_{\text {Salkan }}\right)$ & Saturation coeff. of autotrophs for Alkalinity & 0.5 & $\mathrm{~mol} \mathrm{HCO} / \mathrm{m}^{3}$ & 3 & 0.25 \\
\hline 5 & $\mathrm{~K}_{-}$_оно $_{2}\left(\mathrm{~K}_{\mathrm{f}}\right)$ & Saturation/inhibition coeff. for growth on S_F & 4 & $\mathrm{gCOD} / \mathrm{m}^{3}$ & 3 & 2 \\
\hline 6 & $\mathrm{~K}_{\perp \perp P P \_P A O}\left(\mathrm{~K}_{\mathrm{ipp}}\right)$ & Inhibition coeff. for X_PP storage & 0.02 & gPP/gPAO & 3 & 0.01 \\
\hline 7 & $K_{\text {MAX_fPP_PAO }}\left(K_{\text {mXPp }}\right)$ & Maximum ratio of $X \_P P / X_{P A O}$ & 0.78 & $\mathrm{gPP} / \mathrm{gPAO}$ & 2 & 0.156 \\
\hline 8 & $\mathrm{~K}_{-} \mathrm{S}$ NHHX $_{\mathrm{N}}\left(\mathrm{K}_{\mathrm{Snh}}\right)$ & Saturation coeff. for ammonium (nutrient) & 0.05 & $\mathrm{gN} / \mathrm{m}^{3}$ & 3 & 0.025 \\
\hline 9 & 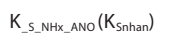 & Saturation coeff. of autotrophs for Ammonium & 1 & $\mathrm{gN} / \mathrm{m}^{3}$ & 3 & 0.5 \\
\hline 10 & $\mathrm{~K}_{\text {_NOX_OHо }}\left(\mathrm{K}_{\text {nохh }}\right)$ & Saturation/inhibition coeff. for nitrate & 0.5 & $\mathrm{gN} / \mathrm{m}^{3}$ & 2 & 0.1 \\
\hline 11 & $\mathrm{~K}_{-02}\left(\mathrm{~K}_{\mathrm{O} 2}\right)$ & Saturation/inhibition coeff. for oxygen & 0.2 & $\mathrm{gO} / \mathrm{m}^{3}$ & 3 & 0.1 \\
\hline 12 & $\mathrm{~K}_{-\mathrm{O} Z \text { _ANO }}\left(\mathrm{K}_{\mathrm{O} 2 \mathrm{an}}\right)$ & Saturation/inhibition coeff. of $\mathrm{X} \_$AUT for $\mathrm{O}_{2}$ & 0.5 & $\mathrm{gO} / \mathrm{m}^{3}$ & 2 & 0.1 \\
\hline 13 & $\mathrm{~K}_{-\mathrm{S} \_\mathrm{PO} 4}\left(\mathrm{~K}_{\mathrm{spo4}}\right)$ & Saturation coeff. for phosphorus (nutrient) & 0.01 & $\mathrm{gP} / \mathrm{m}^{3}$ & 3 & 0.005 \\
\hline 14 & $\mathrm{~K}_{S_{S} \mathrm{PHA}_{-} \text {PAO }}\left(\mathrm{K}_{\text {Spha }}\right)$ & Saturation coeff. for PHA & 0.01 & $\mathrm{gPHA} / \mathrm{gPAO}$ & 3 & 0.005 \\
\hline 15 & $\mathrm{~K}_{\text {S_fPP_PAO_PHA }_{-}}\left(\mathrm{K}_{\text {SpPh }}\right)$ & Saturation coeff. for polyphosphate (X_PP) & 0.01 & gPP/gPAO & 3 & 0.005 \\
\hline 16 & $\mathrm{~K}_{\text {_S_PO4_PAO_PP }}\left(\mathrm{K}_{\text {SpOpp }}\right)$ & Saturation coeff. for phosphorus in X_PP storage & 0.2 & $\mathrm{gP} / \mathrm{m}^{3}$ & 3 & 0.1 \\
\hline 17 & $\mathrm{~K}_{-}$__Blnf_OHO_hyd $_{(}\left(\mathrm{K}_{\text {Sbih }}\right)$ & Saturation coeff. for particulate COD & 0.1 & $\mathrm{gBPO} / \mathrm{gOHO}$ & 3 & 0.05 \\
\hline 18 & 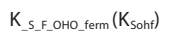 & Saturation coeff. for fermentation on S_F & 20 & $\mathrm{gCOD} / \mathrm{m}^{3}$ & 3 & 10 \\
\hline 19 & $\mathrm{k}_{- \text {M_Blnf_OHO_hyd }}\left(\mathrm{k}_{\text {Mbi }}\right)$ & Hydrolysis rate constant & 4 & $\mathrm{gCOD} /(\mathrm{gCOD} * \mathrm{~d})$ & 3 & 2 \\
\hline 20 & $\mathrm{mu}_{-\mathrm{ANO}}\left(\mathrm{mu}_{\mathrm{ano}}\right)$ & Maximum growth rate of $X \_A U T$ & 1 & $1 / \mathrm{d}$ & 2 & 0.2 \\
\hline 21 & $\mathrm{mu}_{\text {оно }}\left(\mathrm{mu}_{\text {оно }}\right)$ & Maximum growth rate $\mathrm{X} \_\mathrm{OHO}$ on substrate & 6 & $1 / \mathrm{d}$ & 2 & 1.2 \\
\hline 22 & $\mathrm{mu}_{-\mathrm{PAO}}\left(\mathrm{mu}_{\mathrm{pao}}\right)$ & Maximum growth rate for $X \_P A O$ & 1 & $1 / d$ & 2 & 0.2 \\
\hline 23 & $\mathrm{n}_{\text {_No_Het }}\left(\mathrm{n}_{\text {Nhet }}\right)$ & Reduction factor for de-nitrification & 0.3 & - & 2 & 0.06 \\
\hline 24 & $\mathrm{n}_{\text {_oHO_Blinf_hyd }}\left(\mathrm{n}_{\text {Hhihy }}\right)$ & Anoxic hydrolysis reduction factor & 0.6 & - & 2 & 0.12 \\
\hline 25 & $\mathrm{n}_{\text {_оно_Blinf_erm }}\left(\mathrm{n}_{\text {Hhife }}\right)$ & Anaerobic hydrolysis reduction factor & 0.1 & - & 3 & 0.05 \\
\hline 26 & $Q_{\text {PAA_PP_Stor }}\left(\mathrm{Q}_{\mathrm{pph}}\right)$ & Rate constant for storage of X_PHA & 3 & $1 / d$ & 2 & 0.6 \\
\hline 27 & $Q_{\text {_PAO_PO4_PP }}\left(\mathrm{Q}_{\text {popp }}\right)$ & Rate constant for storage of X_PP & 4.5 & $1 / d$ & 2 & 0.9 \\
\hline 28 & $Q_{-}$оно_F_VFA $\left(Q_{\text {fac }}\right)$ & Maximum rate for fermentation & 3 & $1 / \mathrm{d}$ & 2 & 0.6 \\
\hline 29 & $\mathrm{~S}_{-02 \_ \text {sat }}$ & Oxygen saturation concentration & 8.9 & $\mathrm{~g} / \mathrm{m}^{3}$ & 1 & 0.445 \\
\hline 30 & $\mathrm{Y}_{-\mathrm{ANO}}\left(\mathrm{Y}_{\mathrm{ano}}\right)$ & Yield for autotrophic biomass & 0.24 & $\mathrm{gCOD} / \mathrm{gN}$ & 1 & 0.012 \\
\hline 31 & $\mathrm{Y}_{\text {_оно }}\left(\mathrm{Y}_{\text {oho }}\right)$ & Yield for heterotrophic biomass & 0.67 & gCOD/gCOD & 1 & 0.0335 \\
\hline 32 & $Y_{P A O}\left(Y_{\text {paO }}\right)$ & Yield coeff. (biomass/X_PHA) & 0.67 & $\mathrm{gCOD} / \mathrm{gCOD}$ & 1 & 0.0335 \\
\hline 33 & $Y_{\text {Stor_PP_PAO }}\left(Y_{p p}\right)$ & PHA requirement for $X \_P P$ storage & 0.2 & $\mathrm{gCOD} / \mathrm{gP}$ & 1 & 0.01 \\
\hline 34 & $Y_{\text {gly_VFA }}\left(Y_{\text {ppac }}\right)$ & X_PP release as S_PO4 per X_PHA stored & 0.5 & $\mathrm{gP} / \mathrm{gCOD}$ & 1 & 0.02 \\
\hline 35 & $\mathrm{i}_{\mathrm{N} \_ \text {_xBorg_mol_perc }}\left(\mathrm{i}_{\mathrm{n}_{0}}\right)$ & N/C: biodegradable particulate organics & 0.227 & ratio & 3 & 0.1135 \\
\hline 36 & $\mathbf{i}_{-}$_xBlnf_mol_perc $\left(\mathbf{i}_{\mathrm{n} \times \mathrm{bi}}\right)$ & N/C: PS biodegradable particulate organics & 0.033 & ratio & 3 & 0.0165 \\
\hline 37 & $\mathrm{i}_{-}$_xuorg_mol_perc $\left(\mathrm{i}_{\text {nxuo }}\right)$ & N/C: endogenous residue organics & 0.062 & ratio & 3 & 0.031 \\
\hline 38 & $\mathrm{i}_{\text {N__SF_mol_perc }}\left(\mathrm{i}_{\text {nsf }}\right)$ & $\mathrm{N} / \mathrm{C}$ : fermentable biodegradable soluble organics & 0.058 & ratio & 3 & 0.029 \\
\hline 39 & $\mathrm{i}_{\text {NN_org_mol_perc }}\left(\mathrm{i}_{\mathrm{no}}\right)$ & $\mathrm{N} / \mathrm{C}$ : organisms & 0.227 & ratio & 3 & 0.1135 \\
\hline 40 & $\mathrm{i}_{\mathrm{N}_{N} X U \text { Inf_mol_perc }}\left(\mathrm{i}_{\text {nxui }}\right)$ & N/C: unbiodegradable particulate organics & 0.062 & ratio & 3 & 0.031 \\
\hline 41 & $\mathbf{i}_{\text {N__Su_mol_perc }}\left(\mathbf{i}_{\text {nsu }}\right)$ & N/C: unbiodegradable soluble organics & 0.135 & ratio & 3 & 0.0675 \\
\hline 42 & $\mathrm{~b}_{-\mathrm{ANO}}\left(\mathrm{b}_{\mathrm{ano}}\right)$ & Decay rate for $X \_A U T$ & 0.15 & $1 / \mathrm{d}$ & 2 & 0.03 \\
\hline 43 & $\mathrm{~b}_{- \text {оно }}\left(\mathrm{b}_{\text {оно }}\right)$ & Rate constant for lysis and decay for $\mathrm{X} \_\mathrm{OHO}$ & 0.62 & $1 / d$ & 2 & 0.124 \\
\hline 44 & $b_{-P A O}\left(b_{\text {pao }}\right)$ & Rate constant for lysis of X_PAO & 0.04 & $1 / d$ & 2 & 0.008 \\
\hline 45 & $\mathrm{~b}_{-\mathrm{PHA}}\left(\mathrm{b}_{\mathrm{pha}}\right)$ & Rate constant for lysis of X_PHA & 0.04 & $1 / d$ & 2 & 0.008 \\
\hline 46 & $b_{p p p}\left(b_{p p}\right)$ & Rate constant for lysis of X_PP & 0.017 & $1 / \mathrm{d}$ & 2 & 0.0034 \\
\hline 47 & $i_{\text {_p_XBOrg_mol_perc }}\left(\mathrm{i}_{\mathrm{po}}\right)$ & P/C: biodegradable particulate organics & 0.031 & ratio & 3 & 0.0155 \\
\hline 48 & $i_{\text {_p_XBnf_mol_perc }}\left(\mathrm{i}_{\text {pxbi }}\right)$ & P/C: PS biodegradable particulate organics & 0.013 & ratio & 3 & 0.0065 \\
\hline 49 & $\mathrm{i}_{\text {_p_xuorg_mol_perc }}\left(\mathrm{i}_{\mathrm{p} \times \mathrm{u}}\right)$ & $\mathrm{P} / \mathrm{C}:$ endogenous residue organics & 0.012 & ratio & 3 & 0.006 \\
\hline 50 & $i_{\text {_p_SF_mol_perc }}\left(\mathrm{i}_{\text {pst }}\right)$ & P/C: fermentable biodegradable soluble organics & 0.005 & ratio & 3 & 0.0025 \\
\hline 51 & $i_{\text {_p_org_mol_perc }}\left(\mathrm{i}_{\text {po }}\right)$ & $\mathrm{P} / \mathrm{C}$ : organisms & 0.031 & ratio & 3 & 0.0155 \\
\hline 52 & $i_{\_}$_Xulnf_mol_perc $\left(i_{\text {pxui }}\right)$ & P/C: unbiodegradable particulate organics & 0.012 & ratio & 3 & 0.006 \\
\hline 53 & $\mathrm{i}_{\text {_p_su_mol_perc }}\left(\mathrm{i}_{\text {psu }}\right)$ & P/C: unbiodegradable soluble organics & 0.03 & ratio & 3 & 0.015 \\
\hline 54 & $f_{s} s_{-1}$ & Fraction of inert COD in particulate substrate & 0.003 & ratio & 3 & 0.0015 \\
\hline 56 & $f_{-x U \_ \text {Bio_lysis }}\left(f_{x b}\right)$ & Fraction of inert COD generated in biomass lysis & 0.08 & $\mathrm{gCOD} / \mathrm{gCOD}$ & 1 & 0.004 \\
\hline 57 & $\mathbf{i}_{\text {_H_XBOrg_mol_perc }}\left(\mathrm{i}_{\mathrm{H}_{\mathrm{O}}}\right)$ & $\mathrm{H} / \mathrm{C}$ : biodegradable particulate organics & 1.454 & ratio & 3 & 0.727 \\
\hline 58 & $\mathrm{i}_{- \text {H_XBlnf_mol_perc }}\left(\mathrm{i}_{\mathrm{h} \times \mathrm{bi}}\right)$ & H/C: PS biodegradable particulate organics & 2.469 & ratio & 3 & 1.2345 \\
\hline 59 & $i_{\text {_H_Xuorg_mol_perc }}\left(\mathrm{i}_{\text {hxuo }}\right)$ & $\mathrm{H} / \mathrm{C}$ : endogenous residue organics & 1.567 & ratio & 3 & 0.7835 \\
\hline 60 & $i_{\text {_H_SE_mol_perc }}\left(\mathrm{i}_{\text {hsf }}\right)$ & $\mathrm{H} / \mathrm{C}$ : fermentable biodegradable soluble organics & 2.004 & ratio & 3 & 1.002 \\
\hline 61 & $\mathrm{i}_{\text {_H_Org_mol_perc }}\left(\mathrm{i}_{\text {ho }}\right)$ & $\mathrm{H} / \mathrm{C}$ : organisms & 1.454 & ratio & 3 & 0.727 \\
\hline 62 & $i_{-}{ }_{-} X U$ Inf_mol_perc $\left(i_{\text {hxui }}\right)$ & $\mathrm{H} / \mathrm{C}$ : unbiodegradable particulate organics & 1.567 & dUnit/dUnit & 3 & 0.7835 \\
\hline 63 & $\mathrm{i}_{\text {H__SU_mol_perc }}\left(\mathrm{i}_{\mathrm{hsu}}\right)$ & $\mathrm{H} / \mathrm{C}$ : unbiodegradable soluble organics & 1.648 & dUnit/dUnit & 3 & 0.824 \\
\hline 64 & i_o_xBorg_mol_perc $\left(i_{\circ o}\right)$ & $\mathrm{O} / \mathrm{C}$ : biodegradable particulate organics & 0.357 & ratio & 3 & 0.1785 \\
\hline 65 & $\mathrm{i}_{-}$_xBBnf_mol_perc$\left(\mathrm{i}_{\mathrm{oxbi}}\right)$ & O/C: PS biodegradable particulate organics & 0.848 & ratio & 3 & 0.424 \\
\hline 66 & 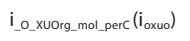 & $\mathrm{O} / \mathrm{C}$ : endogenous residue organics & 0.565 & ratio & 3 & 0.2825 \\
\hline 67 & $\mathrm{i}_{\text {_o_SF_mol_perc }\left(\mathrm{i}_{\text {osf }}\right)}$ & O/C: fermentable biodegradable soluble organics & 0.66 & ratio & 3 & 0.33 \\
\hline 68 & i_o_org_mol_perc $\left(\mathrm{i}_{\text {oo }}\right)$ & $\mathrm{O} / \mathrm{C}:$ organisms & 0.357 & ratio & 3 & 0.1785 \\
\hline 69 & $\mathrm{i}_{\text {_o_XXInf_mol_perc }}\left(\mathrm{i}_{\text {oxui }}\right)$ & O/C: unbiodegradable particulate organics & 0.565 & ratio & 3 & 0.2825 \\
\hline 70 & $\mathrm{i}_{-}$_su_mol_perc $\left(\mathrm{i}_{\text {osu }}\right)$ & O/C: unbiodegradable soluble organics & 0.511 & ratio & 3 & 0.2555 \\
\hline 71 & $i_{\text {_Ca_PP_mol_perp }}\left(\mathrm{i}_{\text {Capp }}\right)$ & $\mathrm{Ca} / \mathrm{P}:$ polyphosphate & 0.053 & ratio & 3 & 0.0265 \\
\hline 72 & $i_{\text {_K_PP_mol_perp }}\left(i_{\text {Kpp }}\right)$ & K/P: polyphosphate & 0.312 & ratio & 3 & 0.156 \\
\hline 73 & $i_{\text {Mg_PP_mol_perp }}\left(\mathrm{i}_{\text {Mgpp }}\right)$ & Mg/P: polyphosphate & 0.297 & ratio & 3 & 0.1485 \\
\hline
\end{tabular}


Table 9. Parameters used in simulating the UCTSDM3P Model

\begin{tabular}{|c|c|c|c|c|c|c|}
\hline No. & Parameter & Description & Initial value & Units & Class & Uncertainty \\
\hline 1 & $\mathrm{~K}_{-\mathrm{CO} 2}$ & Rate constant for $\mathrm{CO}_{2}$ exchange & 0.1 & $1 / \mathrm{d}$ & 3 & 0.05 \\
\hline 2 & $b_{-a c}\left(b_{A c}\right)$ & Decay rate constant for $X \_A C$ & 0.015 & $1 / \mathrm{d}$ & 2 & 0.003 \\
\hline 3 & $b_{-a d}\left(b_{A d}\right)$ & Decay rate constant for $\mathrm{X} \_\mathrm{AD}$ & 0.041 & $1 / d$ & 2 & 0.0082 \\
\hline 4 & $b_{-a m}\left(b_{A m}\right)$ & Decay rate constant for $\mathrm{X} \_A M$ & 0.037 & $1 / d$ & 2 & 0.0074 \\
\hline 5 & $\mathrm{k}_{\mathrm{M} \_ \text {Borg_AD_hyd }}\left(\mathrm{k}_{\mathrm{Mbo}}\right)$ & Half saturation coeff. for WAS BPO hydrolysis & 1.855 & $1 / d$ & 3 & 0.9275 \\
\hline 6 & $\mathrm{k}_{\mathrm{M} \_B \operatorname{lnf} \_A D \_ \text {hyd }}\left(\mathrm{k}_{\mathrm{Mbi}}\right)$ & Half saturation coeff. for PS BPO hydrolysis & 6.797 & $1 / d$ & 3 & 3.3985 \\
\hline 7 & $\mathrm{k}_{\mathrm{H}_{-} \mathrm{F}_{-} \mathrm{A} \_ \text {h } h \mathrm{~d}}\left(\mathrm{k}_{\mathrm{f}}\right)$ & Hydrolysis rate constant for FSO & 10 & $1 / d$ & 3 & 5 \\
\hline 8 & $b_{-h m}\left(b_{H m}\right)$ & Decay rate constant for $\mathrm{X}_{-} \mathrm{HM}$ & 0.01 & $1 / d$ & 2 & 0.002 \\
\hline 9 & $\mathrm{~b}_{- \text {оно_АD }}\left(\mathrm{b}_{\mathrm{oh}}\right)$ & Decay rate constant for $\mathrm{X}_{-} \mathrm{OHO}$ & 13.3 & $1 / d$ & 3 & 6.7 \\
\hline 10 & $\mathrm{~b}_{-P A O \_A D}\left(\mathrm{~b}_{\mathrm{pa}}\right)$ & Decay rate constant for $\mathrm{X} \_\mathrm{PAO}$ & 13.3 & $1 / d$ & 3 & 6.7 \\
\hline 11 & $\mathrm{k}_{\mathrm{H}_{-} \text {PHA_AD_hyd }}\left(\mathrm{b}_{\text {phahyd }}\right)$ & Hydrolysis rate constant for X_PHA & 2 & $1 / d$ & 3 & 1 \\
\hline 12 & $\mathrm{k}_{\mathrm{H}_{-} \mathrm{PP} \_ \text {AD_hyd }}\left(\mathrm{b}_{\text {pphyd }}\right)$ & Hydrolysis rate constant for X_PP & 1 & $1 / d$ & 3 & 0.5 \\
\hline 13 & $\mathrm{k}^{\prime} \mathrm{r}_{\text {cal }}\left(\mathrm{k}_{\mathrm{cal}}\right)$ & Dissolution of calcite & 0.5 & $1 / d$ & 3 & 0.25 \\
\hline 14 & $\mathrm{k}^{\prime} \mathrm{r}_{\text {_cap }}\left(\mathrm{k}_{\text {сар }}\right)$ & Dissolution of calcium phosphate & 350 & $1 / d$ & 3 & 175 \\
\hline 15 & $\mathrm{k}^{\prime} \mathrm{r}_{\text {mag }}\left(\mathrm{k}_{\text {mag }}\right)$ & Dissolution of magnesite & 50 & $1 / d$ & 3 & 25 \\
\hline 16 & $k^{\prime} r_{-m g k p}\left(k_{m g k p}\right)$ & Dissolution of K-struvite & 1000 & $1 / d$ & 3 & 500 \\
\hline 17 & $k^{\prime} r_{-}$newb $\left(k_{\text {newb }}\right)$ & Dissolution of newberyte & 0.05 & $1 / d$ & 3 & 0.025 \\
\hline 18 & $k^{\prime} r_{\text {stru }}\left(k_{\text {stru }}\right)$ & Dissolution of struvite & 2000 & $1 / \mathrm{d}$ & 3 & 1000 \\
\hline 19 & $\mathrm{~K}_{\mathrm{L}-\mathrm{H} 2}\left(\mathrm{~K}_{\mathrm{IH} 2}\right)$ & Inhibition coefficient for $\mathrm{H}_{2}$ in acidogenesis & 1.25 & $\mathrm{~g} / \mathrm{m}^{3}$ & 2 & 0.25 \\
\hline 20 & 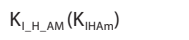 & $\mathrm{H}^{+}$inhibition for acetoclastic methanogens & 0.00000115 & $\mathrm{~mol} / \mathrm{kg}$ & 2 & 0.00000023 \\
\hline 21 & $\mathrm{~K}_{\mathrm{I}_{\perp} \mathrm{H} \text { HM }}\left(\mathrm{K}_{\mathrm{IHH}}\right)$ & $\mathrm{H}^{+}$inhibition for hydrogenotrophic methanogens & 0.00053 & $\mathrm{~mol} / \mathrm{kg}$ & 2 & 0.000106 \\
\hline 22 & $\mathrm{KS}_{\text {-AC }}\left(\mathrm{K}_{\mathrm{SAC}}\right)$ & Half Sat coeff. for acetogens & 6.59 & $\mathrm{~g} / \mathrm{m}^{3}$ & 2 & 1.32 \\
\hline 23 & $\mathrm{KS}_{\text {AD }}\left(\mathrm{K}_{\mathrm{SAd}}\right)$ & Half Sat coeff. for acidogens & 140 & $\mathrm{~g} / \mathrm{m}^{3}$ & 2 & 0.28 \\
\hline 24 & $\mathrm{KS}_{-} \mathrm{AM}\left(\mathrm{K}_{\mathrm{SAm}}\right)$ & Half Sat coeff. for acetoclastic methanogens & 0.78 & $\mathrm{~g} / \mathrm{m}^{3}$ & 2 & 0.156 \\
\hline 25 & $\mathrm{~K}_{\text {S_BOrg_AD_hyd }}\left(\mathrm{K}_{\text {Sbohyd }}\right)$ & Rate constant for WAS BPO hydrolysis & 8.409 & $\mathrm{gCOD} / \mathrm{gCOD}$ & 3 & 4.2045 \\
\hline 26 & $\mathrm{~K}_{\text {S_Blnf_AD_hydd }}\left(\mathrm{K}_{\text {Sbihyd }}\right)$ & Rate constant for PS BPO hydrolysis & 10.829 & $\mathrm{gCOD} / \mathrm{gCOD}$ & 3 & 5.4145 \\
\hline 27 & $\mathrm{Ks}_{\text {_HM }}\left(\mathrm{K}_{\mathrm{Shm}}\right)$ & Half sat. coeff. for X_HM & 0.3145 & $\mathrm{~g} / \mathrm{m}^{3}$ & 2 & 0.0629 \\
\hline 28 & $m u_{-A C}\left(m u_{A C}\right)$ & Max specific growth rate for acetogens (X_AC) & 1.15 & $1 / \mathrm{d}$ & 1 & 0.0575 \\
\hline 29 & $m u_{-A D}\left(m u_{A d}\right)$ & Max specific growth rate for acidogens $\left(X \_A D\right)$ & 0.8 & $1 / d$ & 1 & 0.04 \\
\hline 30 & $\mathrm{mu}_{-A M}\left(\mathrm{mu}_{\mathrm{Am}}\right)$ & Max specific growth rate for $X \_A C$ & 4.39 & $1 / d$ & 1 & 0.2195 \\
\hline 31 & $\mathrm{mu}_{\text {-нм}}\left(\mathrm{mu}_{\mathrm{Hm}}\right)$ & Max specific growth rate for $\mathrm{X} \_\mathrm{HM}$ & 1.2 & $1 / \mathrm{d}$ & 1 & 0.06 \\
\hline 32 & $\mathrm{Y}_{-A C}\left(\mathrm{Y}_{A C}\right)$ & Acidogenesis yield (COD/COD) & 0.0278 & - & 1 & 0.00139 \\
\hline 33 & $Y_{-A D}\left(Y_{A d}\right)$ & Low $\mathrm{H}_{2}$ acetogenesis yield (COD/COD) & 0.1074 & - & 1 & 0.00537 \\
\hline 34 & $Y_{-A H}\left(Y_{A h}\right)$ & High $\mathrm{H}_{2}$ acetogenesis yield (COD/COD) & 0.1074 & - & 1 & 0.00537 \\
\hline 35 & $\mathrm{Y}_{\mathrm{AM}}\left(\mathrm{Y}_{\mathrm{Am}}\right)$ & Acetoclastic methanogenesis yield (COD/COD) & 0.0157 & - & 1 & 0.000785 \\
\hline 36 & $\mathrm{Y}_{\text {-HM }}\left(\mathrm{Y}_{\mathrm{Hm}}\right)$ & Hydrogenotrophic methanogenesis yield & 0.004 & gCOD/gCOD & 1 & 0.0002 \\
\hline 37 & $\mathrm{i}_{\text {_N_XBOrg_mol_perc }}\left(\mathrm{i}_{\mathrm{no}}\right)$ & N/C: biodegradable particulate organics & 0.227 & ratio & 3 & 0.1135 \\
\hline 38 & $\mathrm{i}_{-}{ }_{-} \times \mathrm{B}$ nnf_mol_perc $\left(\mathrm{i}_{\text {nxbi }}\right)$ & N/C: PS biodegradable particulate organics & 0.033 & ratio & 3 & 0.0165 \\
\hline 39 & $\mathrm{i}_{\text {N__xuorg_mol_perc }}\left(\mathrm{i}_{\mathrm{n} \times \mathrm{u}}\right)$ & $\mathrm{N} / \mathrm{C}$ : endogenous residue organics & 0.062 & ratio & 3 & 0.031 \\
\hline 40 & $\mathrm{i}_{\text {N_SE_mol_perc }}\left(\mathrm{i}_{\mathrm{nss}}\right)$ & N/C: fermentable biodegradable soluble organics & 0.058 & ratio & 3 & 0.029 \\
\hline 41 & $\mathrm{i}_{\text {_N_Org_mol_perc }}\left(\mathrm{i}_{\text {no }}\right)$ & $\mathrm{N} / \mathrm{C}$ : organisms & 0.227 & ratio & 3 & 0.1135 \\
\hline 42 & $\mathrm{i}_{-}$_xunn_mol__perc $\left(\mathrm{i}_{\mathrm{n} \times \mathrm{u}}\right)$ & N/C: unbiodegradable particulate organics & 0.062 & ratio & 3 & 0.031 \\
\hline 43 & $i_{\text {N__SU_mol_perc }}\left(\mathbf{i}_{\text {nsu }}\right)$ & N/C: unbiodegradable soluble organics & 0.135 & ratio & 3 & 0.0675 \\
\hline 44 & $i_{\text {_p_xBOrg_mol_perc }}\left(\mathrm{i}_{\mathrm{po}}\right)$ & P/C: biodegradable particulate organics & 0.031 & ratio & 3 & 0.0155 \\
\hline 45 & $i_{\text {_pXXBln_mol_perc }}\left(\mathrm{i}_{\mathrm{pxbi}}\right)$ & P/C: PS biodegradable particulate organics & 0.013 & ratio & 3 & 0.0065 \\
\hline 46 & $\mathrm{i}_{\text {_pxxuorg_mol_perc }}\left(\mathrm{i}_{\text {pxuo }}\right)$ & P/C: endogenous residue organics & 0.012 & ratio & 3 & 0.006 \\
\hline 47 & $\mathrm{i}_{\text {P___E__mol_perc }}\left(\mathrm{i}_{\text {pst }}\right)$ & P/C: fermentable biodegradable soluble organics & 0.005 & ratio & 3 & 0.0025 \\
\hline 48 & $\mathrm{i}_{\text {_p_org_mol_perc }}\left(\mathrm{i}_{\mathrm{po}}\right)$ & $\mathrm{P} / \mathrm{C}:$ organisms & 0.031 & ratio & 3 & 0.0155 \\
\hline 49 & $\mathrm{i}_{-}$_xulnf_mol_perc $\left(\mathrm{i}_{\text {pxui }}\right)$ & P/C: unbiodegradable particulate organics & 0.012 & ratio & 3 & 0.006 \\
\hline 50 & $i_{\text {_p_su_mol_perc }}\left(\mathrm{i}_{\text {psu }}\right)$ & P/C: unbiodegradable soluble organics & 0.03 & ratio & 3 & 0.015 \\
\hline 51 & $f_{\text {XU_Bio_lysis }}\left(f_{x u b}\right)$ & Fraction of dead biomass to endogenous residue & 0.08 & ratio & 1 & 0.004 \\
\hline 52 & $\mathrm{k}_{M_{-} \text {FP_PAO_PHA }}\left(\mathrm{k}_{\text {MPpha }}\right)$ & Rate constant for $X \_P P$ release & 5 & $1 / \mathrm{d}$ & 3 & 2.5 \\
\hline 53 & ISS_Bм $\left(f_{\text {xio }}\right)$ & ISS to biomass for X_OHO and X_PAO & 0.15 & $g / g C O D$ & 1 & 0.0075 \\
\hline 54 & $i_{\text {_H_XBOrg_mol_perc }}\left(\mathrm{i}_{\mathrm{ho}}\right)$ & $\mathrm{H} / \mathrm{C}$ : biodegradable particulate organics & 1.454 & ratio & 3 & 0.727 \\
\hline 55 & 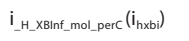 & H/C: PS biodegradable particulate organics & 2.469 & ratio & 3 & 1.2345 \\
\hline 56 & $\mathrm{i}_{\text {-H_Xuorg_mol_perc }}\left(\mathrm{i}_{\mathrm{h} \times \text { ×uo }}\right)$ & $\mathrm{H} / \mathrm{C}$ : endogenous residue organics & 1.567 & ratio & 3 & 0.7835 \\
\hline 57 & $\mathrm{i}_{\text {H__SF_mol_perc }}\left(\mathrm{i}_{\text {hsf }}\right)$ & H/C: fermentable biodegradable soluble organics & 2.004 & ratio & 3 & 1.002 \\
\hline 58 & $\mathrm{i}_{\text {_H_Org_mol_perc }}\left(\mathrm{i}_{\text {ho }}\right)$ & $\mathrm{H} / \mathrm{C}$ : organisms & 1.454 & ratio & 3 & 0.727 \\
\hline 59 & $\mathbf{i}_{-}{ }_{-} x$ Unf_mol_perc $\left(\mathbf{i}_{\text {hxui }}\right)$ & $\mathrm{H} / \mathrm{C}$ : unbiodegradable particulate organics & 1.567 & ratio & 3 & 0.7835 \\
\hline 60 & $\mathrm{i}_{\text {_H_SU_mol_perc }}\left(\mathrm{i}_{\text {hsu }}\right)$ & H/C: unbiodegradable soluble organics & 1.648 & ratio & 3 & 0.824 \\
\hline 61 & $\mathrm{i}_{\text {_o_xBOrg_mol_perc }}\left(\mathrm{i}_{\mathrm{o}} \textrm{ }\right)$ & $\mathrm{O} / \mathrm{C}$ : biodegradable particulate organics & 0.357 & ratio & 3 & 0.1785 \\
\hline 62 & i_o_xBlnf_mol_perc $\left(\mathrm{i}_{\mathrm{oxbb}}\right)$ & O/C: PS biodegradable particulate organics & 0.848 & ratio & 3 & 0.424 \\
\hline 63 & i_o_xuorg_mol_perc $\left(\mathrm{i}_{\text {oxuo }}\right)$ & $\mathrm{O} / \mathrm{C}:$ endogenous residue organics & 0.565 & ratio & 3 & 0.2825 \\
\hline 64 & $\mathrm{i}_{\text {_o_SF_mol_perc }}\left(\mathrm{i}_{\text {osf }}\right)$ & O/C: fermentable biodegradable soluble organics & 0.66 & ratio & 3 & 0.33 \\
\hline 65 & $\mathrm{i}_{\text {_o_org_mol_perc }}\left(\mathrm{i}_{\text {oo }}\right)$ & $\mathrm{O} / \mathrm{C}$ : organisms & 0.357 & ratio & 3 & 0.1785 \\
\hline 66 & i__oxulnf_mol_perc $\left(\mathrm{i}_{\text {oxui }}\right)$ & $\mathrm{O} / \mathrm{C}$ : unbiodegradable particulate organics & 0.565 & ratio & 3 & 0.2825 \\
\hline 67 & $\mathrm{i}_{\text {_o_su_mol_perc }}\left(\mathrm{i}_{\text {osu }}\right)$ & O/C: unbiodegradable soluble organics & 0.511 & ratio & 3 & 0.2555 \\
\hline 68 & $\mathrm{i}_{\text {_Ca_PP_mol_perp }}\left(\mathrm{i}_{\text {Capp }}\right)$ & $\mathrm{Ca} / \mathrm{P}:$ polyphosphate & 0.053 & ratio & 3 & 0.0265 \\
\hline 69 & 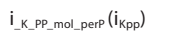 & K/P: polyphosphate & 0.312 & ratio & 3 & 0.156 \\
\hline 70 & $i_{\text {Mg_PP_mol_perp }}\left(\mathrm{i}_{\text {Mgpp }}\right)$ & Mg/P: polyphosphate & 0.297 & ratio & 3 & 0.1485 \\
\hline
\end{tabular}




\section{SENSITIVITY ANALYSIS}

The importance of sensitivity analysis in model calibration is prompted by the notable limitation in the applicability of various WWTP dynamic models, based on the complexities brought about by wide ranges of parameters and the intricate dependence of output variables on these parameters and other state variables. For simpler steady-state models (i.e., those of Wentzel et al. (1990) for BEPR AS systems and and Sötemann et al. (2005); Ekama (2009); Ikumi (2011) for AD systems) the identification of major stoichiometric parameters could be identified intuitively, since these models contain explicit equations linking parameters to output variables. However, the more complex dynamic models are based on differential equations, for prediction of output variables due to changing material loads and flows. The performance of a complete sensitivity analysis on the dynamic model allowed for assement of both linear and/or non-linear effects of all the model parameters on the output variables.

Two sensitivity analysis methods were applied in this study i.e., Morris screening (screening method) and standardised regression coefficients (based on regression). The application of multiple sensitivity analysis methods with multiple objectives was done as recommended by Neumann (2012), as this is expected to lead to more robust conclusions. The results obtained using these methods were used to identify (i) important parameters that would cause a significant change in model outputs, and hence need to be known well, (ii) non-influential parameters (those that can be set to any value within their range without much change in outputs) and (iii) interacting parameters (Neumann, 2012).

To initiate the sensitivity analysis process, uncertainty propagation was conducted by a Monte Carlo (MC) simulation of the model by random sampling of parameter values. The parameter value ranges (i.e., lower ( $\theta$ i_min) and upper ( $\theta$ i_max) prior bounds for the MC simulation) were chosen according to the method proposed by Brun et al. (2002). These parameters were assumed to be uniformly distributed within their ranges. A 1000 simulations were performed using the WEST software (Vanhooren et al., 2003), with 1000 sets of random parameter values generated in this way, to provide 1000 sets of values for the selected output variables, which could then be visualised as histograms or density distributions or characterised in terms of descriptive statistics.

\section{Standard regression coefficient method}

The standard regression coefficients $\left(\mathrm{SRC}_{\mathrm{ij}}\right)$ due to each parameter quantify the effect on variable $j$ when parameter $i$ is changed (hence allows prioritisation of important parameters). The SRC method involves the fitting of a multivariate linear model to the output of the MC simulation (Martin et al., 2010; Neumann et al., 2012). The SRC's multivariate linear regressions relate each output variable $\left(y_{j}\right)$ to all uncertainty parameters $\left(\theta_{j}\right)$, to get an equation of the form:

$$
y_{j}(\theta)=b_{j 0}+\sum_{i=1}^{r} b_{i j} \cdot \theta_{i}
$$

The standard regression coefficient is defined as

$$
S R C_{i j}=\beta_{i j}=b_{i j} \cdot \frac{\sigma_{y j}}{\sigma_{\theta i}}
$$

where $b_{i}$ is the slope obtained from linear regression; $\sigma_{\theta i j}$ is the standard deviation of the 1000 parameter values generated for parameter $I$, and $\sigma_{y j}$ is the resulting standard deviation of output variable $y_{j}$. Finally, the coefficient of determination $\left(R^{2}\right)$, that indicates how well the multilinear regression model fits the variable's responses, was also calculated using the $\mathrm{R}$ program (R Development Core Team, 2011). This indicates how much confidence can be placed in using the calculated values in predicting future results. For variables with $R^{2}>0.7$, the SRCs $\left(\beta_{i}\right)$ are a valid measure of sensitivity (Saltelli et al., 2004).

\section{Morris screening method}

Morris's screening method (Morris, 1992) is a method used to determine elementary effects for each parameter, to identify which parameters affect the model output variables significantly, and to eliminate non-influential parameters. The computation of these elementary effects requires the variation of one parameter at a time (OAT) across a select number of $k$ levels (in this case 10), requiring $k \cdot r$ simulations (where $r$ is the number of parameters). In this design, each model parameter is varied within a selected uncertainty range of $p$, which is also determined using the method proposed by Brun et al. (2002). While a particular parameter was varied, all others were assigned their mid-range values. The elementary effect of parameter $\theta_{i}$ on variable $y_{j}$ is calculated as:

$$
d_{i j}\left(\theta_{i}\right)=\frac{\left[y_{j}\left(\theta_{i}, \ldots, \theta_{i-1}, \theta, \theta_{i}+\Delta, \theta_{i+1}, \ldots, \theta_{r}\right)-y_{j}(\theta)\right]}{\Delta}
$$

where $\mathrm{y}(\theta)$ is the output variable obtained when all parameters are set to their prior values, i.e., $y\left(\theta_{1}, \theta_{2} \ldots \theta_{r}\right)$.

The mean $\left(\mu_{i j}\right)$ and standard deviation $\left(\sigma_{i j}\right)$ of the calculated $k$ elementary effects are determined for each parameter as measures of the parameter importance. $\mu_{i j}$ is used to detect parameters with an important overall influence on the output, while $\sigma_{i j}$ is used to detect parameters involved in interaction with other parameters or whose effect is non-linear (Neumann, 2012; Campolongo et al., 2007).

\section{RESULTS}

Neumann (2012) proposes interpretation of sensitivity analysis results through considering the parameters that are most important (which would cause significant changes in model outputs, hence are a priority to be known), and non-influential (hence can be placed anywhere within their uncertainty range without incurring much of a change). In the following sensitivity analysis, the parameters are grouped into those that are kinetic (hence affect the process rates) and those that are non-kinetic (mainly used in determination of the input component characteristics (e.g., the $\mathrm{X}, \mathrm{Y}, \mathrm{Z}, \mathrm{A}$ and $\mathrm{B}$ values of biomass elemental composition $\mathrm{C}_{\mathrm{X}} \mathrm{H}_{\mathrm{Y}} \mathrm{O}_{Z} \mathrm{~N}_{\mathrm{A}} \mathrm{P}_{\mathrm{B}}$ ) or yield for $\mathrm{OHO}$ biomass growth, $\left.\mathrm{Y}_{\mathrm{H}}\right)$ ).

Table 10 shows the standardised regression coefficients (SRC, $\beta_{i j}$ ) and resulting coefficient of determination for the most important parameters (having the greatest SRC magnitudes) of selected output variables, from the ASM2-3P and UCTSDM3P models. The SRCs are taken to be a valid measure of sensitivity as long as the resulting coefficient of determination, $R^{2}$, is greater than 0.7 (Saltelli et al., 2004). However, lower degrees of linearity indicate that the SRC is being used outside the application range, which could cause the underestimation of important parameters, hence cannot offer a useful contribution towards the estimates of parameter prioritisation (Neumann, 2012). Additional to SRC data, the results from the Morris screening method are also presented - by plotting the expectancy $\left(\mu^{*}\right)$ of the absolute values of the elementary effects against the standard deviation $\sigma$ of the elementary effects for each parameter.

\section{Activated sludge (AS) systems}

In this section the results from the sensitivity analysis of the PWM_SA model parameters, when used to simulate the NDBEPR UCT system (see Fig. 1), are presented.

Biological $\mathrm{P}$ removal involves (i) the anaerobic utilisation of volatile fatty acids to form PHB, which occurs with polyphosphate release to OP, and (ii) the aerobic breakdown of PHB for PAO growth and PP synthesis and uptake. The various parameters identified to be of importance using the SRC and Morris screening sensitivity analysis techniques for the relevant bio $\mathrm{P}$ removal model predicted outputs are discussed below. 
Table 10. Summary of results for PWM_SA (ASM2-3P and UCTSDM3P) uncertainty and sensitivity analysis using SRC method

\begin{tabular}{|c|c|c|c|c|c|c|c|c|c|c|}
\hline \multirow{3}{*}{$\begin{array}{l}\text { Model } \\
\text { ASM2-3P }\end{array}$} & \multirow{3}{*}{$\begin{array}{l}\text { Unit } \\
\text { Anaerobic }\end{array}$} & \multirow{3}{*}{$\begin{array}{l}\text { Variable } \\
\text { FSO (an_SF) }\end{array}$} & \multicolumn{4}{|c|}{ Standard regression coefficient $(\beta)$} & \multirow{3}{*}{$\begin{array}{c}R^{2} \\
0.86\end{array}$} & \multirow{3}{*}{$\begin{array}{c}\begin{array}{c}\text { Kinetic } \\
\text { sum }\left(\mathrm{SRC}^{2}\right)\end{array} \\
0.38\end{array}$} & \multirow{3}{*}{$\begin{array}{c}\begin{array}{c}\text { Stoich. } \\
\text { sum }\left(\mathrm{SRC}^{2}\right)\end{array} \\
0.45\end{array}$} & \multirow{3}{*}{$\begin{array}{c}\begin{array}{c}\text { Total sum } \\
\left(\mathrm{SRC}^{2}\right)\end{array} \\
0.83\end{array}$} \\
\hline & & & \multicolumn{2}{|c|}{ Most positive } & \multicolumn{2}{|c|}{ Most negative } & & & & \\
\hline & & & $i_{\text {osf }}$ & 0.57 & $\mathrm{Q}_{\mathrm{fac}}$ & -0.42 & & & & \\
\hline & Zone & Nitrates (an_Nox) & $\mathrm{K}_{\text {noxh }}$ & 0.37 & $\mathrm{~K}_{\text {Sohf }}$ & -0.29 & 0.74 & 0.39 & 0.33 & 0.72 \\
\hline & & Orthophosphates (an_OP) & $f_{\text {sbsa }}$ & 0.87 & $\mathrm{f}_{\text {Sup }}$ & -0.26 & 0.99 & 0.05 & 0.93 & 0.98 \\
\hline & & Acetate (an_Ac) & $K_{\mathrm{sac}}$ & 0.61 & $\mathrm{Q}_{\mathrm{pph}}$ & -0.69 & 0.90 & 0.87 & 0.08 & 0.95 \\
\hline & & Poly-phosphates (an_PP) & $K_{\text {mxpp }}$ & 0.47 & $\mathrm{i}_{\mathrm{ho}}$ & -0.30 & 0.96 & 0.35 & 0.59 & 0.94 \\
\hline & & Poly-hydroxy-alkanoates (an_PHA) & $f_{\text {sbsa }}$ & 0.85 & $f_{\text {sup }}$ & -0.26 & 0.91 & 0.06 & 0.84 & 0.90 \\
\hline & Anoxic & Ammonia $\left(\mathrm{ax} \_\mathrm{NH}_{4}\right)$ & $i_{\text {no }}$ & 0.17 & $m u_{a n o}$ & -0.42 & 0.41 & 0.29 & 0.11 & 0.40 \\
\hline & Zone & Nitrates $\left(\mathrm{ax} \_\mathrm{NO}_{3}\right)$ & $i_{\text {nxbi }}$ & 0.44 & $i_{\text {hxbi }}$ & -0.29 & 0.80 & 0.30 & 0.47 & 0.77 \\
\hline & & $\mathrm{pH}\left(\mathrm{ax} \_\mathrm{pH}\right)$ & $i_{\mathrm{hxbi}}$ & 0.72 & $i_{\text {oxb }} i$ & -0.37 & 0.97 & 0.04 & 0.98 & 1.02 \\
\hline & Aerobic & Calcium (ae_Ca) & $i_{\mathrm{ho}}$ & 0.18 & $\mathrm{i}_{\text {Capp }}$ & -0.75 & 0.96 & 0.13 & 0.82 & 0.95 \\
\hline & Zone & Potassium (ae_K) & $i_{\mathrm{ho}}$ & 0.19 & $\mathrm{i}_{\mathrm{Kpp}}$ & -0.73 & 0.96 & 0.14 & 0.80 & 0.94 \\
\hline & & Magnesium (ae_Mg) & $i_{\text {ho }}$ & 0.18 & $\mathrm{i}_{\text {Mgpp }}$ & -0.71 & 0.95 & 0.12 & 0.77 & 0.89 \\
\hline & & Ammonia (ae_NH) & $i_{\text {nxbi }}$ & 0.35 & $m u_{a n o}$ & -0.49 & 0.69 & 0.37 & 0.31 & 0.68 \\
\hline & & Nitrates (ae_Nox) & $i_{\text {nxbi }}$ & 0.64 & $i_{\text {no }}$ & -0.41 & 0.81 & 0.07 & 0.83 & 0.90 \\
\hline & & Ortho-phosphates (ae_OP) & $i_{\mathrm{ho}}$ & 0.28 & $\mathrm{f}_{\text {Sbsa }}$ & -0.48 & 0.97 & 0.23 & 0.75 & 0.98 \\
\hline & & Autotrophic nitrifiers (ae_ANO) & $i_{\text {nxbi }}$ & 0.64 & $i_{n o}$ & -0.43 & 0.93 & 0.07 & 0.87 & 0.94 \\
\hline & & Ordinary heterotrophic organisms (ae_OHO) & $Y_{\text {oho }}$ & 0.28 & $f_{\text {Sbsa }}$ & -0.50 & 0.95 & 0.35 & 0.58 & 0.92 \\
\hline & & Phosphorus accumulating organisms (ae_PAO) & $f_{\text {sbsa }}$ & 0.60 & $\mathrm{f}_{\text {sup }}$ & -0.28 & 0.98 & 0.19 & 0.67 & 0.86 \\
\hline & & Polyphosphate (ae_PP) & $f_{\text {sbsa }}$ & 0.44 & $i_{\text {ho }}$ & -0.28 & 0.96 & 0.32 & 0.62 & 0.94 \\
\hline & & Poly-hydroxy-alkanoate (aePHA) & $\mathrm{K}_{\text {Spha }}$ & 0.21 & $\mathrm{mu}_{\text {pao }}$ & -0.14 & 0.17 & 0.11 & 0.07 & 0.19 \\
\hline & & Oxygen utilisation rate (OUR) & $i_{\text {nxbi }}$ & 0.30 & $\mathrm{f}_{\text {Sup }}$ & -0.63 & 0.95 & 0.08 & 0.87 & 0.94 \\
\hline & & Volatile settleable solids (VSS) & $\mathrm{K}_{\text {Snhan }}$ & 0.17 & $m u_{\text {ano }}$ & -0.49 & 0.35 & 1.02 & 1.10 & 2.12 \\
\hline \multirow[t]{16}{*}{ UCTSDM3P } & Anaerobic & Methane $\left(\mathrm{CH}_{4}\right)$ & $k_{\text {Mbohyd }}$ & 0.33 & $\mathrm{~K}_{\text {sbohyd }}$ & -0.70 & 0.96 & 0.60 & 0.34 & 0.94 \\
\hline & Digester & Calcium (S_Ca) & $i_{\text {capp }}$ & 0.42 & $i_{n o}$ & -0.70 & 0.87 & 0.02 & 0.87 & 0.89 \\
\hline & $(A D)$ & Potassium (S_K) & $i_{\mathrm{kpp}}$ & 1.00 & $\mathrm{i}_{\text {Mgpp }}$ & -0.08 & 1.00 & 0.00 & 1.00 & 1.00 \\
\hline & & Magnesium (S_Mg) & $i_{\text {Mgpp }}$ & 0.62 & $i_{\text {no }}$ & -0.68 & 0.91 & 0.01 & 0.92 & 0.92 \\
\hline & & Ammonia (S_NH) & $i_{\mathrm{no}}$ & 0.94 & $\mathrm{i}_{\text {ho }}$ & -0.18 & 0.98 & 0.03 & 0.97 & 1.00 \\
\hline & & Phosphates $\left(\mathrm{S}_{-} \mathrm{PO}_{4}\right)$ & $i_{\mathrm{po}}$ & 0.49 & $\mathrm{i}_{\text {no }}$ & -0.69 & 0.92 & 0.00 & 0.94 & 0.94 \\
\hline & & Biodegradable particulate organics (XBOrg) & $K_{\text {sbohyd }}$ & 0.69 & $\mathrm{k}_{\text {Mbohyd }}$ & -0.34 & 0.95 & 0.59 & 0.32 & 0.91 \\
\hline & & Newberryte (X_Newb) & $i_{\text {Mgpp }}$ & 0.49 & $i_{\text {no }}$ & -0.29 & 0.49 & 0.12 & 0.36 & 0.49 \\
\hline & & Polyphosphate (X_PP) & $i_{\text {psu }}$ & 0.01 & $k_{\text {pphyd }}$ & -1.00 & 0.99 & 0.99 & 0.00 & 0.99 \\
\hline & & Poly-hydroxy-butyrate (X_PHB) & $i_{\mathrm{ho}}$ & 0.03 & $\mathrm{k}_{\text {phahyd }}$ & -0.93 & 0.87 & 0.88 & 0.00 & 0.88 \\
\hline & & Struvite (X_Struv) & $i_{\text {no }}$ & 0.86 & $i_{\mathrm{oo}}$ & -0.22 & 0.86 & 0.01 & 0.86 & 0.88 \\
\hline & & Calcium phosphate (X_ACP) & $i_{\text {no }}$ & 0.65 & $\mathrm{i}_{\text {Mgpp }}$ & -0.26 & 0.89 & 0.02 & 0.90 & 0.91 \\
\hline & & Cabonate alkalinity (CO3 Alk) & $i_{\text {no }}$ & 0.86 & $\mathrm{i}_{\mathrm{ho}}$ & -0.34 & 0.96 & 0.02 & 0.96 & 0.98 \\
\hline & & Chemical oxygen demand (COD) & $i_{\text {hxuo }}$ & 0.39 & $\mathrm{i}_{\text {oxui }}$ & -0.77 & 0.96 & 0.05 & 0.92 & 0.97 \\
\hline & & Inorganic settleable solids (ISS) & $i_{\text {по }}$ & 0.86 & $\mathrm{i}_{\text {oo }}$ & -0.22 & 0.87 & 0.01 & 0.87 & 0.89 \\
\hline & & $\mathrm{pH}$ & $i_{\text {ho }}$ & 0.56 & $i_{o o}$ & -0.30 & 0.81 & 0.02 & 0.84 & 0.86 \\
\hline
\end{tabular}

These include (i) biomass growth and oxygen utilisation due to organic removal, (ii) ammonia utilisation and nitrate generation by autotrophic nitrifying organisms (ANOs), and (iii) bio-P removal through PP accumulation.

\section{Breakdown of organics for biomass growth and oxygen utilisation}

The UCT plant-wide model (PWM_SA) defines the characteristics of sewage biodegradable organics by parameterising the molar fraction elements from its given generic stoichiometric formula (i.e. $\mathrm{X}, \mathrm{Y}, \mathrm{Z}, \mathrm{A}, \mathrm{B}$ in $\mathrm{C}_{\mathrm{X}} \mathrm{H}_{\mathrm{Y}} \mathrm{O}_{\mathrm{Z}} \mathrm{N}_{\mathrm{A}} \mathrm{P}_{\mathrm{B}}$ ). During simulations the mass-balanced stoichiometric processes are then used to track the energy, materials $(\mathrm{C}, \mathrm{H}, \mathrm{O}, \mathrm{N}$ and $\mathrm{P})$ and charge towards prediction of the unit process outputs. The energy (COD) and the nutrients ( $\mathrm{N}$ and $\mathrm{P}$ ) bound in biodegradable organics are biologically utilised in the reactors, while those in the unbiodegradable organics remain conserved (without participating in the biochemical reactions) and accumulate in the system with the solid (for particulate unbiodegradables, UPO) and liquid retention times (for particulate unbiodegradables, UPO, and soluble unbiodegradables, USO, respectively). The volatile fatty acids (VFAs, sourced from the influent or generated through anaerobic fermentation) play a significant role in bio- $\mathrm{P}$ removal, as they are taken up by PAOs as their source of substrate. It can be noticed from Figs 2 and 3 that the fractionation of influent waste and determination of the fraction of influent COD that is unbiodegradable particulate $\left(f_{\text {Sup }}\right)$ and VFA $\left(f_{\text {sbsa }}\right)$ are very important parameters that influence the growth of biomass (hence sludge generation) and oxygen utilisation in the AS system.

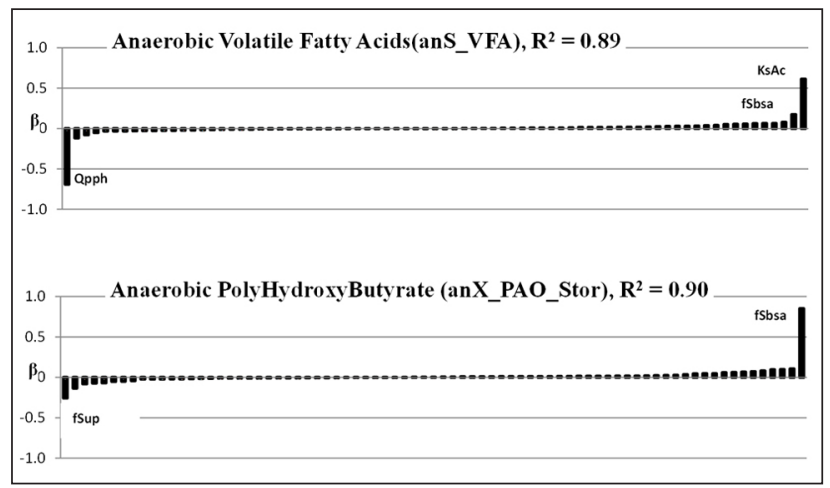

Figure 2. SRC results for sensitivity analysis of anaerobic variables S_VFA and X_PAO_Stor (PHB) to ASM2-3P parameters 


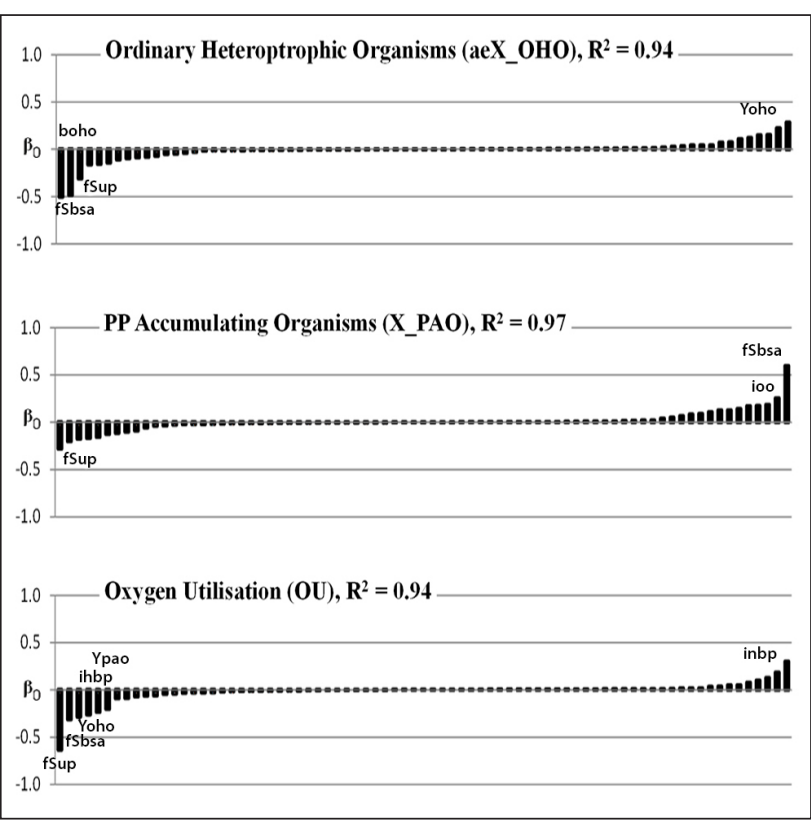

Figure 3. SRC results for sensitivity analysis of aerobic variables $\mathrm{X} \_\mathrm{OHO}, \mathrm{X} \_\mathrm{PAO}$ and oxygen utilisation rate (OU) to ASM2-3P parameters

For the reactor VFA concentration, the SRC method exhibits that $\mathrm{Ks}_{\mathrm{AC}}$ (i.e., the saturation/inhibition coefficient for acetate utilisation) and $Q_{\mathrm{pph}}$ (i.e., the rate constant for storage of poly $\beta$-hydroxybutyrate (PHB)) are also significant parameters (Fig. 2). The PAOs rely mostly on anaerobic uptake of readily biodegradable material for growth (i.e., fermentable biodegradable soluble organics (FBSO) and VFAs). These readily biodegradable organics are converted to an energy storage compound (poly3hydroxy-butyrate, PHB) that is later used aerobically for growth and polyphosphate storage (Wentzel et al., 1990). By dictating the rate of PHB uptake, $Q_{p p h}$ significantly influences the quantity of substrate allocated for growth of PAOs, with the remainder of the biodegradable organics mainly apportioned to the OHOs. This substrate allocation, of course, depends on the availability of VFAs (that are present in the influent or are generated through anaerobic fermentation FBSOs), for conversion to PHB. Intuitively, it is also expected that the $Y_{\text {pao }}$ (yield coefficient for utilisation of PHB in PAO biomass growth) would have a significant positive influence on the predicted reactor PAO population, since it dictates the substrate allocation for anabolic utilisation of PHB in the aerobic zone of the AS system. This is not clearly reflected by the SRC results (Fig. 3) but can be noted by the Morris screening results (Fig. 4), which show $Y_{\text {pao }}$ with the greatest influence and $m u_{\text {pao }}$ (the maximum specific growth rate of PAOs) with the highest degree of non-linearity.
The OHO biomass has various parameters of importance, as indicated by Fig. 3. This includes the kinetic parameter $b_{\text {oho }}$ (i.e., the rate constant for lysis and decay of $\mathrm{OHO}$ biomass) and stoichiometric parameter $Y_{\text {oho }}$ (i.e., the yield coefficient for $\mathrm{OHO}$ biomass growth). The Morris screening method also indicates $Y_{\text {oho }}$ to be the significant parameter, with $\mathrm{mu}_{\mathrm{pao}}$ (the maximum specific growth rate of $\mathrm{OHOs}$ ) having the highest degree of nonlinearity (Fig. 4). The parameters that contribute to biomass elemental formulation (i.e., $i_{\text {no }} i_{\text {ho }}, i_{\text {oo }}$ and $i_{\text {no }}$ - the $\mathrm{H}, \mathrm{O}$ and $\mathrm{N}$ molar content in biomass elemental formula, respectively) are also expected to be significant because their values will determine the electron-donating capacity of the biomass, hence their electron requirements to carry out their metabolic processes. The fraction of energy allocated from breakdown of biodegradable organics to build up biomass cells depends on the biomass yield (i.e., $Y_{\text {oho }}$ ), hence the notable positive impact of this parameter. The $b_{\text {oho }}$ is shown to have a significant (though negative) influence on reactor $\mathrm{OHO}$ concentration because it determines rate of OHO biomass death and degradation (hence a high $b_{\text {oho }}$ value would result in low biomass population, for a given system sludge age). The $Q_{\mathrm{pph}}$ is also expected to have a negative influence here because the biodegradable organics in the influent that are taken up by PAOs are deducted from the organics utilisable by OHOs (the configuration of NDBEPR systems allows for the influent to be exposed to the anaerobic zone prior to aerobic, ensuring that the readily biodegradables are first available for sequestering by PAOs before the OHOs can utilise them aerobically - this allows the PAOs the competitive advantage required for them to co-exist with OHOs as mixed cultures in the NDEBPR AS system). The capability of the model to replicate this system behaviour was evaluated by Ikumi et al. (2015) by applying the PWM_SA model to a Modified Ludzack Ettinger (MLE) system, whereby in this case no PAO growth (hence no PP storage) occurred. However, the same MLE system with little or no nitrification (the unaerated zone now anaerobic) exhibits growth of PAOs. Likewise, sufficiently high quantities of nitrate being recycled to the anaerobic reactor of an $\mathrm{N}$ and P removal system would supress EBPR, as observed during winter in 3 and 5-stage Bardenpho systems, when denitrification is lower (Ikumi, 2011)

Both the SRC and Morris screening methods indicate that the biomass yield ( $Y_{\text {oho }}$ and $Y_{\text {pao }}$ ) parameters have a significant influence on oxygen utilisation. The SRC results also show that the elemental composition of the biodegradable organics (i.e., $i_{\text {nbp }}$ $i_{\mathrm{hbp}}, i_{\mathrm{obp}}$ - the $\mathrm{H}, \mathrm{O}$ and $\mathrm{N}$ molar content in biodegradable organics elemental formulae, respectively) to be significant. This is expected because the biomass yield values dictate the proportion of substrate (biodegradable organics) electrons that are allocated to biomass, with the remainder apportioned to oxygen for generation of energy (hence the negative influence with SRC).

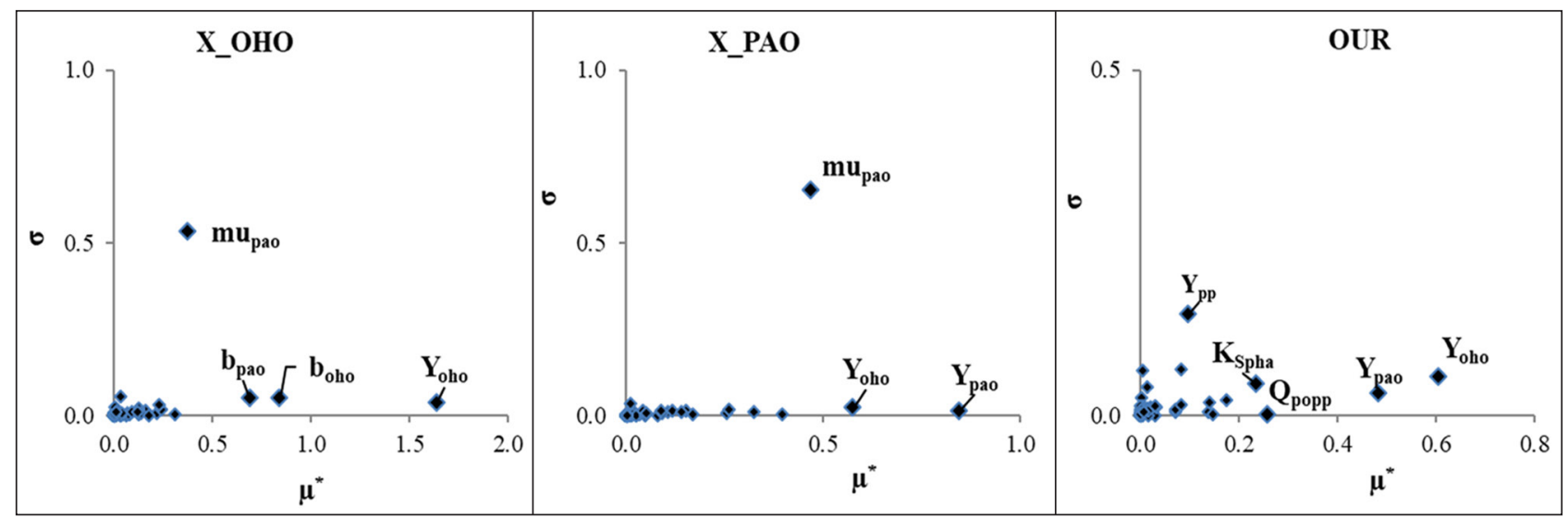

Figure 4. Morris screening results for sensitivity analysis of AS system biomass (X_OHO, and X_PAO) and oxygen utilisation rate (OU) to ASM2-3P parameters 


\section{Utilisation of ammonia}

According to the SRC method, the most significant parameters for prediction of effluent ammonia $\left(\mathrm{NH}_{4}\right)$ concentration include $\mathrm{Ks}_{\text {Nhan }}$ (i.e., the saturation coefficient of autotrophs for ammonium), $\mathrm{mu}_{\mathrm{ano}}$ (i.e., maximum growth rate of autotrophic nitrifying organisms) and $i_{\text {no }}$ (See Fig. 5). This is expected because the $i_{\text {no }}$ dictates the nitrogen requirement, to be sourced from the pool of ammonia in the reactor, for biomass growth. In the activated sludge (AS) models, the ammonia use gives priority to its use as a nutrient during anabolism of faster growing biomass (OHO and $\mathrm{PAO}$ ) before the 'excess' ammonia is used

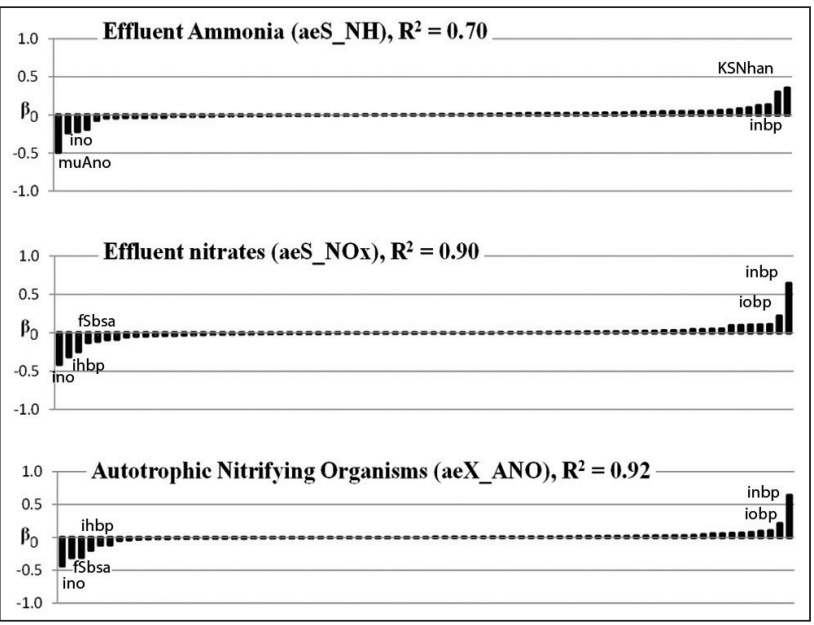

Figure 5. SRC results for sensitivity analysis of ammonia (S_NH), nitrates (S_NOx) and ANO biomass (X_ANO) to ASM2-3P parameters

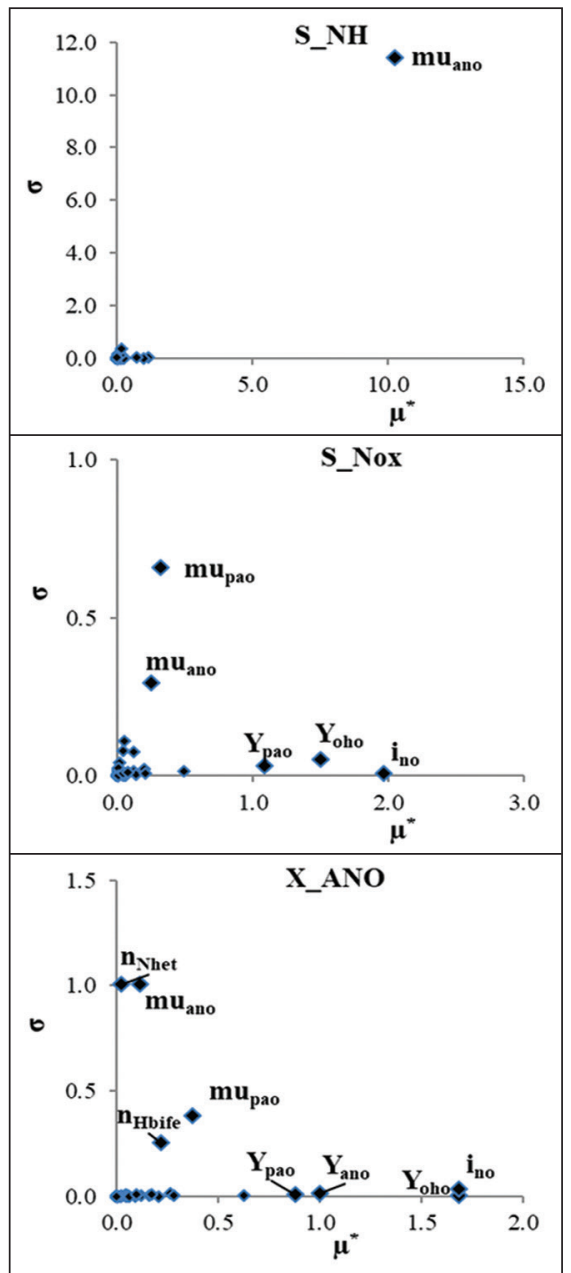

Figure 6. Morris screening results for sensitivity analysis of ammonia ( $\mathrm{S}$ $\mathrm{NH}$ ), nitrates (S_NOx) and ANO biomass (X_ANO) to ASM2-3P parameters for nitrification (the nitrifying organisms that use the ammonia as e-donor are slower growing microorganisms). This is possibly also the reason for $i_{\text {no }}$ being a significant parameter for effluent nitrate $\left(\mathrm{NO}_{3}{ }^{-}\right)$concentrations. Despite this, the ammonia used for nitrification is usually higher than that for biomass growth; hence the parameters that drive the kinetics of this process $\left(\mathrm{mu}_{\mathrm{ano}}\right.$ and $\left.\mathrm{Ks}_{\mathrm{Nhan}}\right)$ have a significant influence. Similar to ammonia and nitrates, the ANO biomass is influenced by $i_{\text {no }}$ and $b_{\text {ano }}$ (Fig. 5). Moreover, the fraction of influent COD as VFA $\left(f_{\text {sbsa }}\right)$, together with the elemental composition of the biodegradable particulate organics (notable by the significance of $i_{\text {nbp }}, i_{\text {hbp }}, i_{\text {obp }}$ parameters), are influential towards ANO growth and reactor nitrate concentration.

The Morris screening results indicate similar parameters as being of significance to SRC $\left(\mathrm{mu}_{\text {ano, }}\right.$, has a high $\mu^{*}$ value and also a relatively significant degree of non-linearity for ammonia concentration. For the ANO growth and reactor nitrate concentration, $\mathrm{mu}_{\mathrm{ano}}$ appears to have more of a non-linear influence, but still a relatively low $\mu^{*}$ value. However, also similar to SRC, the results show that $i_{\text {no }}$ is the most important parameter for these variables. Further notable parameters influencing ANO growth and reactor nitrate concentration are $Y_{\text {oho }}$ and $Y_{\text {pao }}$ (Fig. 6). This is possible because the increased growth of $\mathrm{OHO}$ and $\mathrm{PAO}$ biomass would result in a greater $\mathrm{N}$ requirement as nutrient source, especially if the $i_{\text {no }}$ value is high.

\section{Biological phosphorus removal}

Figures 7 and 8 indicate the parameters of significance for $\mathrm{P}$ removal via aerobic PP uptake and prediction of effluent OP concentration. According to the SRC method of sensitivity analysis, the most important parameters for OP are $k_{\text {mxpp }}$ (i.e., the maximum polyphosphate (PP) content of PAO biomass), $f_{\text {Sbsa }}, i_{\text {pbp }}$ (the P molar content in biodegradable organics elemental formula) and elemental composition of biomass (dictated by notable $i_{\mathrm{ho}}, i_{\mathrm{no}}$ and $i_{\mathrm{oo}}$ parameters). From the ASM2-3P model (Ikumi et al., 2015), PHB is aerobically utilised for PAO growth and for storage of PP. The $k_{\text {mxpp }}$ parameter dictates the quantity of reactor OP to be utilised for PP formation, for each new PAO biomass formed (with sufficient PHB available aerobically, higher $k_{\text {mxpp }}$ results in lower effluent OP). The $i_{\text {pbp }}$ informs the quantity of $\mathrm{P}$ that could be released as $\mathrm{OP}$ from biodegradable particulates; hence - apart from the influent OP - act as a significant source of reactor $\mathrm{OP}$ that could be used in this process.

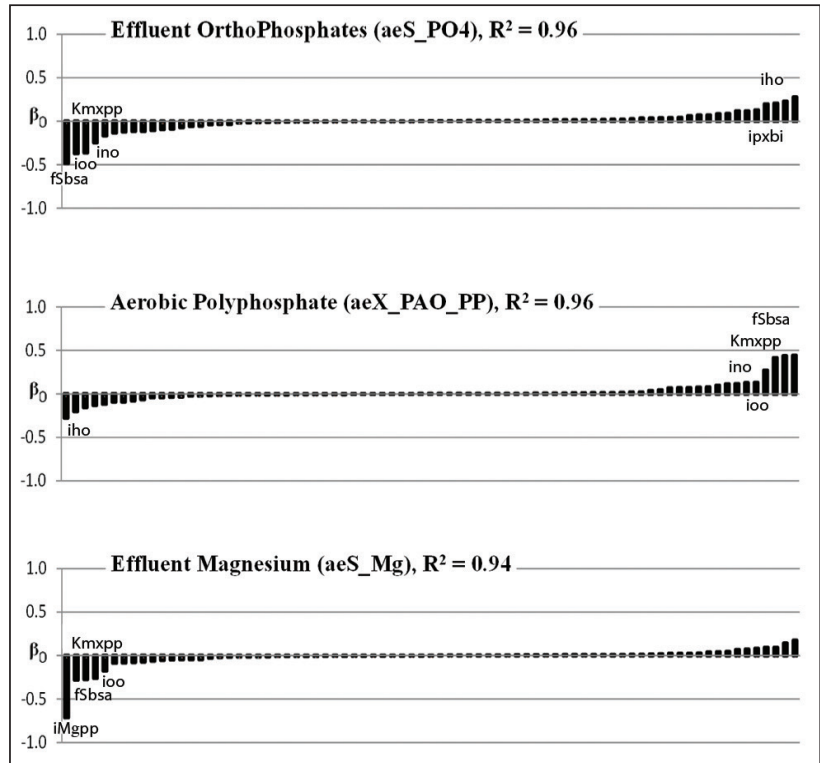

Figure 7. SRC results for sensitivity analysis of phosphates (S_PO4), Poly-P (X_PAO_PP) and magnesium (S_Mg) to ASM2-3P parameters 


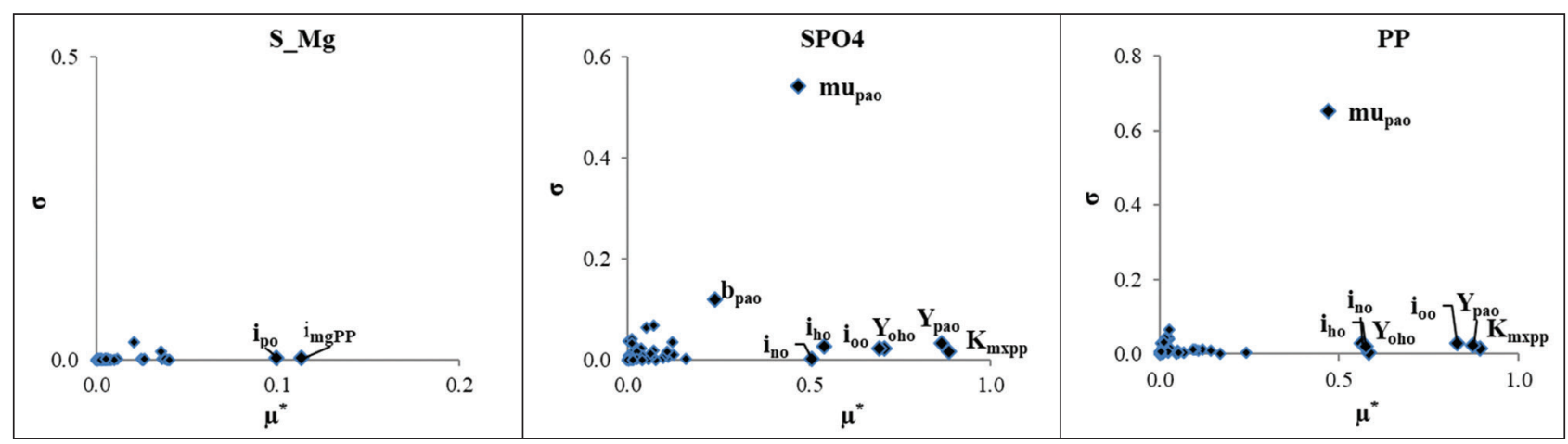

Figure 8. Morris screening results for sensitivity analysis of phosphates (S_PO4), Poly-P (X_PAO_PP) and magnesium (S_Mg) to ASM2-3P parameters

Since most of the OP used aerobically is utilised for the generation of PP (the OP taken up for biomass growth and released in biomass endogenous death is usually much lower), it can also be observed that the same parameters that are significant to OP also impact $\mathrm{PP}$, but in the opposite way (PP increase results in OP decrease so the parameters that would have a negative effect on the formation of PP would have a positive effect on concentration of OP; see Fig. 7). Moreover, because $\mathrm{Mg}$ is also a crucial component to be utilised in generation of PP, similar parameters influencing OP concentration $\left(f_{\text {sbsa }}, K_{\text {mxpp }}\right.$ and $\left.i_{\text {oo }}\right)$ are noted to be important for prediction of effluent $\mathrm{Mg}$ concentration. The only difference with $\mathrm{Mg}$ is that $i_{\text {mgpp }}$ (i.e., the $\mathrm{Mg}$ molar content in polyphosphate) is an added significant parameter for aerobic concentration of $\mathrm{Mg}$. An increase in $i_{\text {mgpp }}$ indicates that more $\mathrm{Mg}$ is required for PP formation, which would result in decreased concentration of effluent Mg. This is observed in both the SRC and Morris screening method (see Figs 7 and 8 ) where $i_{\text {mgpp }}$ has the highest $\mu^{*}$ value (i.e., is the most important). Also, similar to SRC, the results from the Morris screening method also indicate $K_{\text {mxpp' }}$, $i_{\text {ho, }}, i_{\text {no }}$ and $i_{\text {oo }}$ to be significant parameters for aerobic PP and OP concentrations. However, other parameters of importance are the biomass yield values (i.e., $Y_{\text {oho }}$ and $Y_{\text {pao }}$ with high $\mu^{*}$ values) and $\mathrm{mu}_{\mathrm{pao}}$ (with the highest degree of non-linearity, $\sigma$ ).

\section{Anaerobic digestion unit process}

In simulating the AD of sludge generated (from PS and NDBEPR WAS) with the UCTSDM3P model, the selected output variables to be applied during the sensitivity analysis were those considered to be indicative of system performance and resource recovery. These variables included residual biodegradable organics (BPO), methane $\left(\mathrm{CH}_{4}\right)$, ammonia $\left(\mathrm{NH}_{4}{ }^{+}\right)$, ortho-phosphates $\left(\mathrm{HPO}_{4}{ }^{2-}\right.$ and $\mathrm{H}_{2} \mathrm{PO}_{4}^{-}$), metals ( $\mathrm{Mg}, \mathrm{K}$ and $\mathrm{Ca}$ ), precipitates (mainly struvite), alkalinity (for carbonate, $\mathrm{H}_{2} \mathrm{CO}_{3}{ }^{*}$ Alk. and phosphate, $\mathrm{H}_{3} \mathrm{PO}_{4}$ Alk., weak acid/base systems) and the system $\mathrm{pH}$. The $\mathrm{BPO}$ removal and methane generation is associated with energy recovery potential; the prediction of low system $\mathrm{pH}$ indicates a warning for system failure and the aqueous phase products (e.g., $\mathrm{NH}_{4}^{+}$, OP, $\mathrm{Mg}, \mathrm{K}$ and $\mathrm{Ca}$ ) are later generated in the dewatering liquor that would either be recycled upstream or transferred to side-stream treatment processes.

\section{BPO removal}

The kinetics of hydrolysis of biomass BPO is usually the slowest process in $\mathrm{AD}$ and ends up determining the residual $\mathrm{BPO}$ and the nutrients released in the process, contributing towards final $\mathrm{AD}$ products. From Fig. 9, it can be noticed that for the WAS biomass $\mathrm{BPO}$, the most important parameters are $\mathrm{Ks}_{\text {bohyd }}$ and $\mathrm{kM}_{\text {bohyd }}$ (i.e., the half saturation coefficient for hydrolysis of biomass BPO and the maximum specifichydrolysis rate constant for WASbiomass $\mathrm{BPO}$, respectively). These are the hydrolysis rate kinetic constants that drive the breakdown of biomass BPO. The Morris screening results agree with the SRC, by showing the same hydrolysis kinetic constants to be most influential (see Fig. 10). For the AD of PS, the $\mathrm{Ks}_{\text {bihyd }}$ and $\mathrm{kM}_{\text {bihyd }}$ are also important for PS BPO removal (Fig. 11). However, the unbiodegradable fraction of PS COD $\left(f_{\text {sup }}\right)$ is also significant (especially notable in the case of $\mathrm{CH}_{4}$ evolution), since it significantly impacts the quantity of substrate available for conversion to biogas (where, since soluble influent organics are acceptably of low concentrations, the BPO COD available for conversion to $\mathrm{CH}_{4} \mathrm{COD}$ is mainly $\left(1-f_{\text {sup }}\right)$ of PS COD).

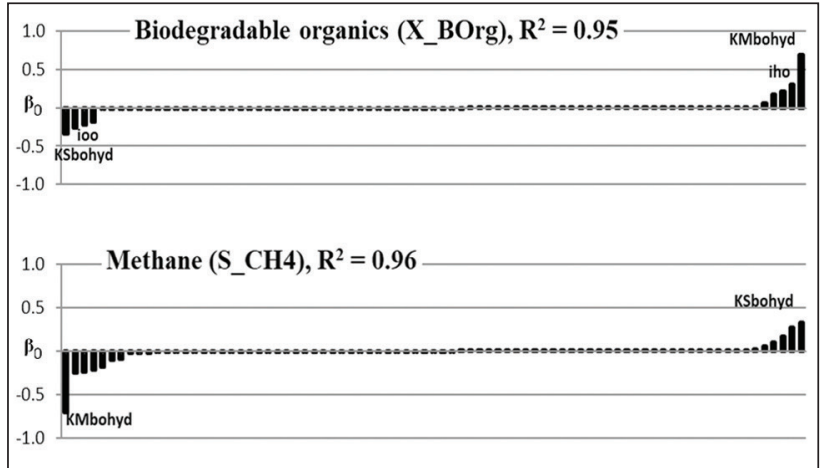

Figure 9. SRC results for sensitivity analysis of biomass biodegradable organics (X_BOrg) and methane generation (S_CH4) to UCTSDM3P parameters for EBPR WAS digestion

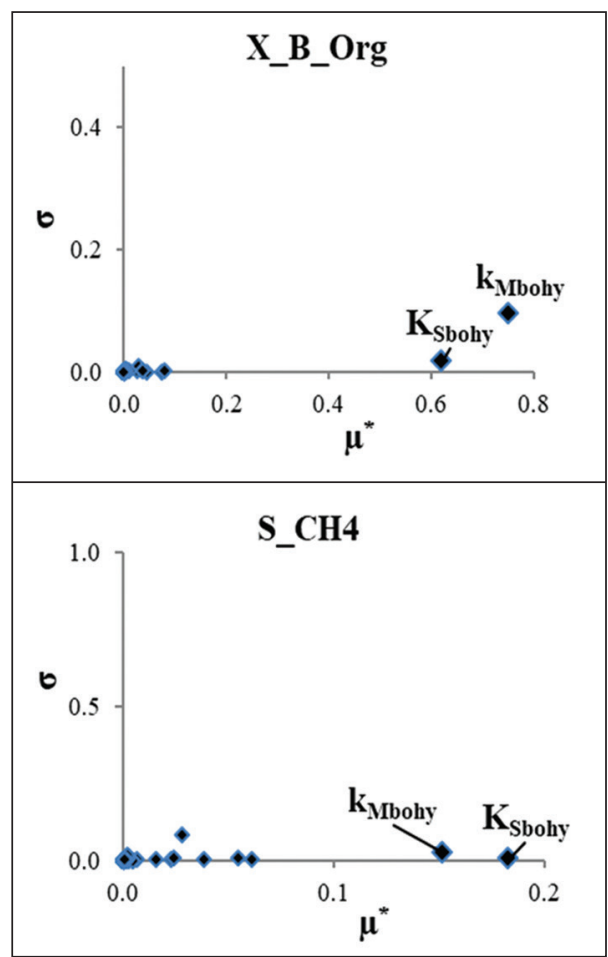

Figure 10. Morris screening results for sensitivity analysis of biomass biodegradable organics (X_BOrg) and methane generation (S_CH4) to UCTSDM3P parameters for EBPR WAS digestion 


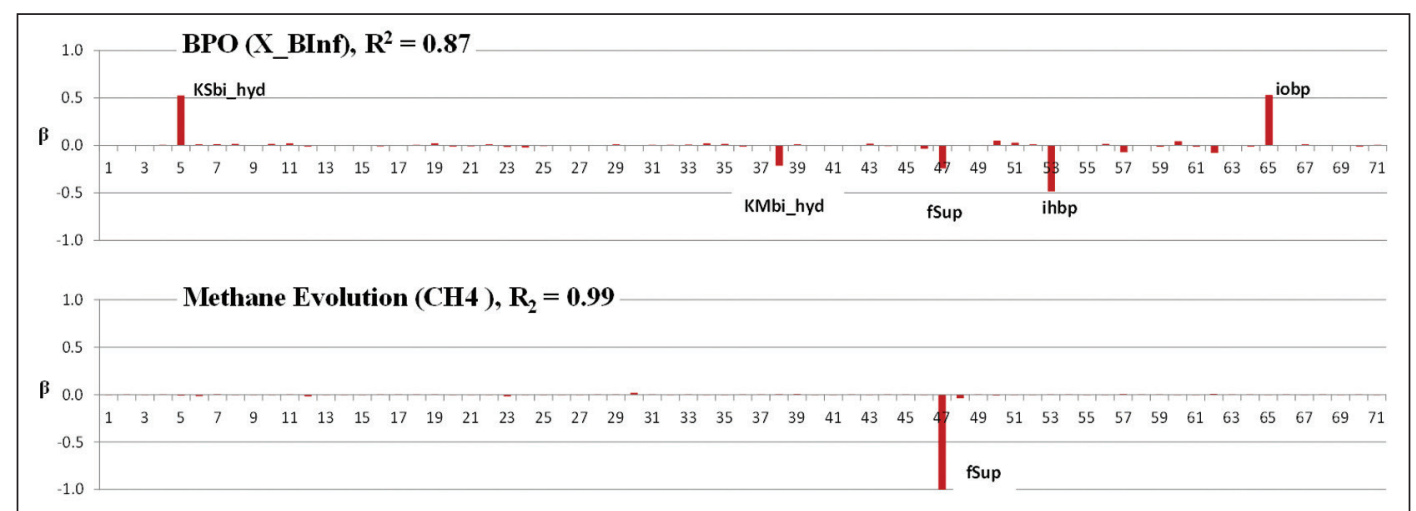

Figure 11. SRC results for sensitivity analysis of Influent biodegradable particulate organics (X_BInf) and methane generation (S_CH4) to UCTSDM3P parameters for PS digestion

\section{Ammonia release and associated alkalinity change}

From Fig. 12, it can be noticed that for modelling ammonia $\left(\mathrm{NH}_{4}\right)$ releases in WAS $\mathrm{AD}$, the most significant parameter is $i_{\mathrm{no}}$, and equivalently $i_{\text {nbp }}$, for hydrolysis of PS. These parameters are depicted to have a positive influence on ammonia generation (Figs 12 and 13, its higher values would result in increased ammonia concentration). The results are intuitively adequate, because the $A$ value from $\mathrm{C}_{\mathrm{X}} \mathrm{H}_{\mathrm{Y}} \mathrm{O}_{\mathrm{Z}} \mathrm{N}_{\mathrm{A}} \mathrm{P}_{\mathrm{B}}$ of $\mathrm{BPO}$ (parameterised in the UCTSDM3P model as $i_{\text {no }}$ for biomass BPO and $i_{\text {nbp }}$ for $\mathrm{PS}$ ) dictates the ammonia to be released into the $\mathrm{AD}$ from the $\mathrm{N}$ bound in hydrolysed BPO. The PS additionally has $f_{\text {Sup }}, i_{\text {hbp }}$ and $i_{\text {obp }}$ as influential parameters.

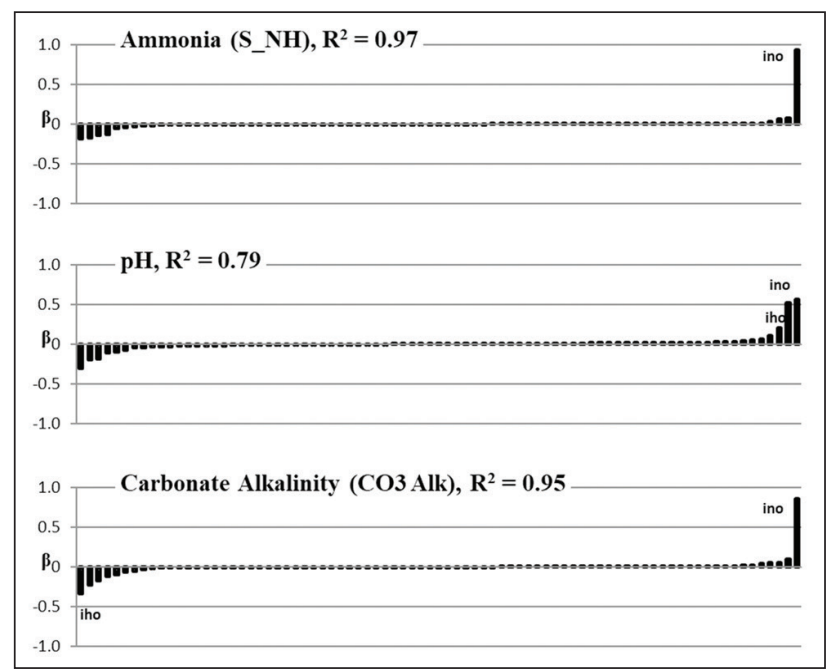

Figure 12. SRC results for sensitivity analysis of ammonia (S_NH) and pH and $\mathrm{H}_{2} \mathrm{CO}_{3}$ alkalinity to UCTSDM3P parameters for EBPR WAS digestion
The results from the Morris screening method (Fig. 14) also agree with the SRC data (which can be accepted due to the high $R^{2}$ value of 0.97 ) on the importance of $i_{\text {no }}$ in $\mathrm{NH}_{4}$ release prediction. According to the SRC method of analysis, the same parameter that had a strong influence on $\mathrm{NH}_{4}$ release (i.e., $i_{\text {no }}$ ) has a significant impact on the sensitivity of the system $\mathrm{pH}$ and carbonate alkalinity. For mixed weak acid/base systems controlled by the inorganic carbon system, bicarbonate $\left(\mathrm{HCO}_{3}^{-}\right)$production is the main generator of $\mathrm{H}_{2} \mathrm{CO}_{3}{ }^{*}$ alkalinity (i.e., $\mathrm{H}_{2} \mathrm{CO}_{3}{ }^{*}$ alkalinity $\approx\left[\mathrm{HCO}_{3}{ }^{-}\right]$; Ikumi et al., 2015) and the establishment of system $\mathrm{pH}$. According to the weak acid/base equilibrium formulations (Loewenthal et al., 1994), the $\left[\mathrm{CO}_{3}{ }^{2-}\right]$ is a relatively very small species of the carbonate system in the steady-state methanogenic $\mathrm{AD} \mathrm{pH}$ ranges (around 6.5 to 8 ). Hence, the $\mathrm{AD}$ is modelled such that the stoichiometric products that assist in uptake of $\mathrm{H}^{+}$from dissolved $\mathrm{CO}_{2}\left(\mathrm{H}_{2} \mathrm{CO}_{3}{ }^{*}\right)$ are the main factors that promote increase in $\mathrm{H}_{2} \mathrm{CO}_{3}{ }^{*} \mathrm{Alk}$, hence also increasing the $\mathrm{pH}\left(-\log \left[\mathrm{H}^{+}\right]\right)$. The organically bound $\mathrm{N}$ is modelled to be released as $\mathrm{NH}_{3}$ (non-ionic form of ammonia, that is a non-reference species for the ammonia weak acid/base system), which picks up this $\mathrm{H}^{+}$from $\mathrm{H}_{2} \mathrm{CO}_{3}{ }^{*}$ of the inorganic carbon (IC) system forming $\mathrm{HCO}_{3}$. This results in the ammonia releases from organic $\mathrm{N}$ causing an increase in alkalinity generation and hence increased system $\mathrm{pH}$. However, for the $\mathrm{pH}$ and $\mathrm{H}_{2} \mathrm{CO}_{3}{ }^{*} \mathrm{Alk}$ variables, the Morris screening method does not reflect the same results as SRC but instead indicates the $Y_{\text {ppac }}$ (the yield value for acetate uptake during anaerobic PP release) as the most significant parameter. However, these results are also possible because in $\mathrm{AD}$ fed P-rich sludge, the PP release process has a significant impact on the system $\mathrm{pH}$ (see section below). For P-rich systems with PP, the aqueous $\mathrm{H}_{2} \mathrm{CO}_{3}{ }^{*}$ alkalinity increase also depends on PP and cellbound $\mathrm{P}$ release because $\mathrm{PP}$ is released $\mathrm{H}_{2} \mathrm{PO}_{4}$ and biomass $\mathrm{P}$ is released as $\mathrm{H}_{3} \mathrm{PO}_{4}$, which interact with the other weak acid/base systems and influence $\mathrm{pH}$.

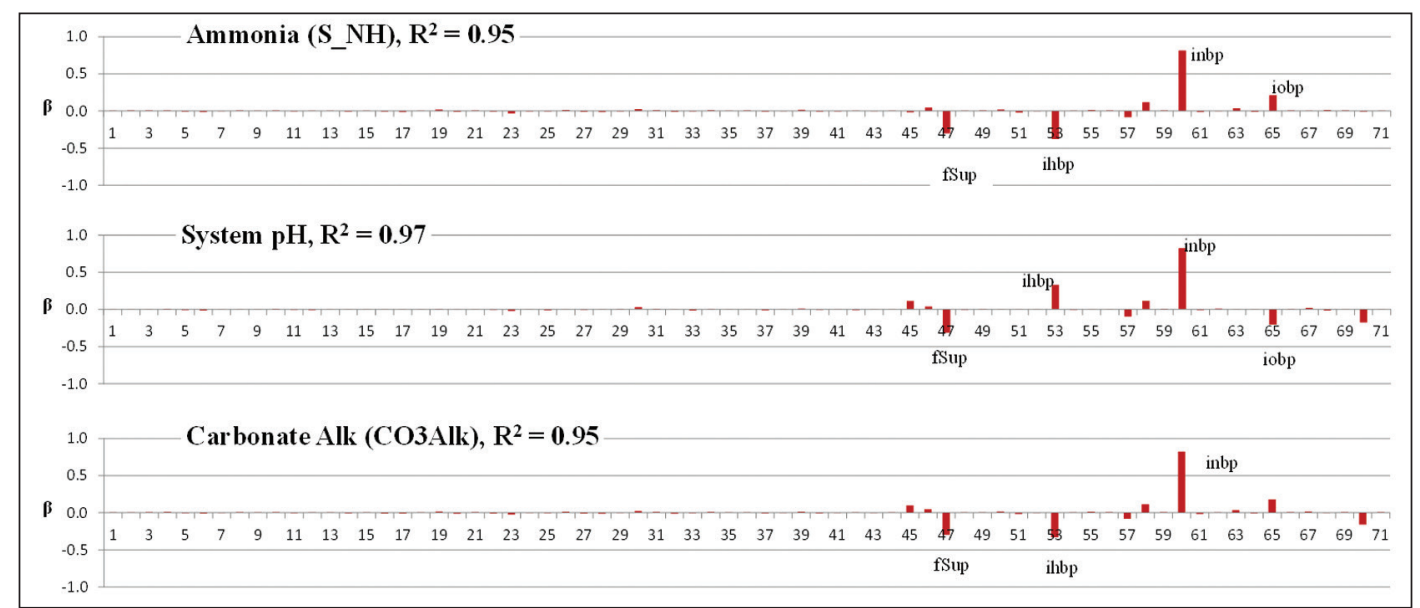

Figure 13. SRC results for sensitivity analysis of ammonia (S_NH) and $\mathrm{pH}$ and $\mathrm{H}_{2} \mathrm{CO}_{3}$ alkalinity to UCTSDM3P parameters for PS digestion 


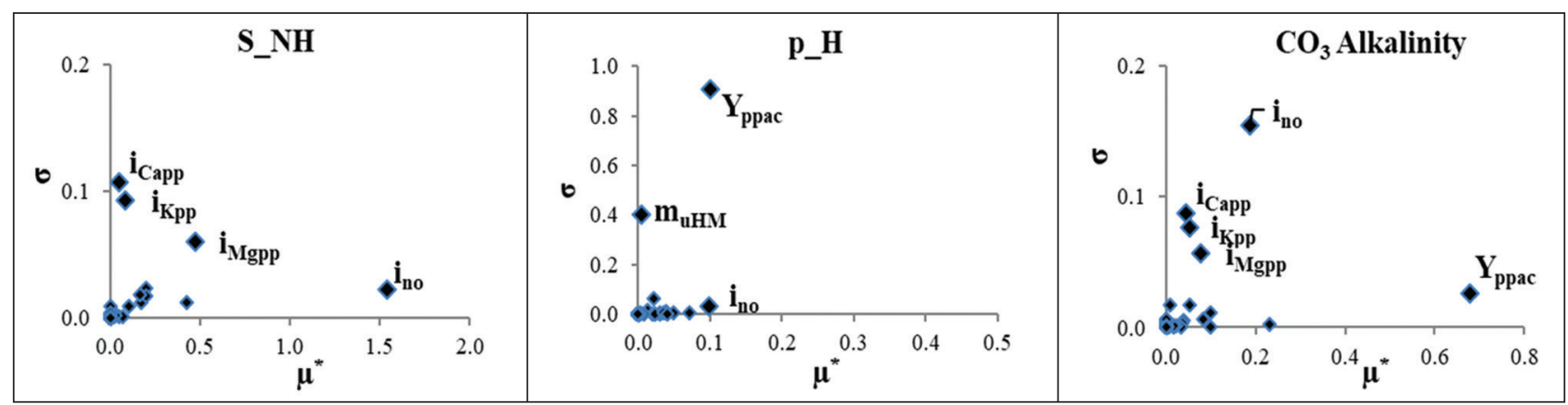

Figure 14. Morris screening results for sensitivity analysis of ammonia (S_NH) and $\mathrm{pH}$ and $\mathrm{H}_{2} \mathrm{CO}_{3}$ alkalinity to UCTSDM3P parameters for EBPRWAS digestion

\section{Orthophosphate release and struvite precipitation potential}

The four main forms of $\mathrm{P}$ in AD include organically bound P, PP, OP and precipitate $\mathrm{P}$. The release of OP from breakdown of NDBEPR WAS in AD has been noted to impact the system alkalinity and mineral precipitation potential (Ikumi et al., 2015). Figure 15 shows the most important parameter for orthophosphate concentration in the AD effluent is $i_{\text {no }}$ followed by $i_{\mathrm{po}}$. The influence of $i_{\text {no }}$ on AD effluent OP is possible because: (i) increase in ammonia release with BPO (WAS biomass) breakdown causes increase in alkalinity and $\mathrm{pH}$ (as discussed the section above), and (ii) ammonia is a component part of struvite $\left(\mathrm{MgNH}_{4} \mathrm{PO}_{4}\right)$, hence influences struvite precipitation potential (this precipitation requires $\mathrm{OP}$ uptake).

It is expected that most of the $\mathrm{OP}$ generated in the $\mathrm{AD}$ aqueous phase is due to the occurrence of PP breakdown. This PP breakdown process is modelled in a similar way in $\mathrm{AD}$ to that in the anaerobic reactor of the parent AS system - i.e., most of the PP is released immediately to generate energy for synthesis of PHA from acetate (Harding et al., 2010; Ikumi, 2011). This is because the environmental requirements for this process (i.e., sufficient quantities of acetate, from fermentation of biodegradable organics and the lack of an external terminal electron acceptor) are also present in $\mathrm{AD}$ reactors. However, after all $\mathrm{PP}$ is rapidly broken down in the $\mathrm{AD}$ ( $\mathrm{PP}$ - i.e., $\mathrm{Mg}_{\mathrm{c}} \mathrm{K}_{\mathrm{d}} \mathrm{Ca}_{\mathrm{e}} \mathrm{PO}_{3}$, breakdown results in release of its constituent $\mathrm{Mg}, \mathrm{K}, \mathrm{Ca}$ and $\mathrm{OP}$ ), there is ample supply of $\mathrm{OP}$ and some $\mathrm{Mg}$ in the aqueous phase of the $\mathrm{AD}$ reactor. This, together with the release of $\mathrm{NH}_{4}{ }^{+}$, which occurs with $\mathrm{BPO}$ hydrolysis, increases the precipitation potential of struvite $\left(\mathrm{MgNH}_{4} \mathrm{PO}_{4}\right)$ - when the concentration of the ions contributing to formation of the struvite mineral (i.e., $\mathrm{Mg}, \mathrm{NH}_{4}{ }^{+}$and $\mathrm{PO}_{4}{ }^{3-}$ ) are significantly high. Because struvite precipitation is encouraged by higher $\mathrm{pH}$, the increased alkalinity associated with higher $\mathrm{N}$ releases from BPO (mainly due to the high $i_{\text {no }}$ values) further encourages struvite precipitation. The struvite precipitation in turn uses OP, resulting in reduction in OP present in the aqueous phase of $\mathrm{AD}$ mixed liquor, hence the negative impact of $i_{\text {no }}$ on OP (i.e., OP decreases with increase in $i_{\text {no }}$ ). Conversely, the $i_{\mathrm{po}}$ represents the amount of $\mathrm{P}$ bound to the biodegradable organic material entering the $\mathrm{AD}$, hence dictates the amount of $\mathrm{OP}$ to eventually be released with complete utilisation of these biodegradable organics. The $\mathrm{P}$ bound to biodegradable organics is much smaller than that in $\mathrm{PP}$ and gets released at a much slower rate (with the degradation of biodegradable organic material). Hence, relative to PP the organically bound $\mathrm{P}$ is not expected to have a major impact on $\mathrm{OP}$ released, although it does contribute to the total OP present in the $\mathrm{AD}$ system and add to the precipitation potential of struvite.

The Morris screening analysis results (Fig. 16) show that $i_{\text {mgpp }}$ is the important parameter influencing $\mathrm{OP}$ concentration in AD. At longer sludge ages, where almost all the ammonia is released, the Mg (most made available from PP release and hydrolysis) is usually the component (out of $\mathrm{Mg}, \mathrm{OP}$ and $\mathrm{NH}_{4}$, which form struvite) with the lowest concentration. Hence, it usually plays a role as the limiting factor for struvite precipitation (i.e., struvite precipitation comes to a stop after Mg gets depleted).

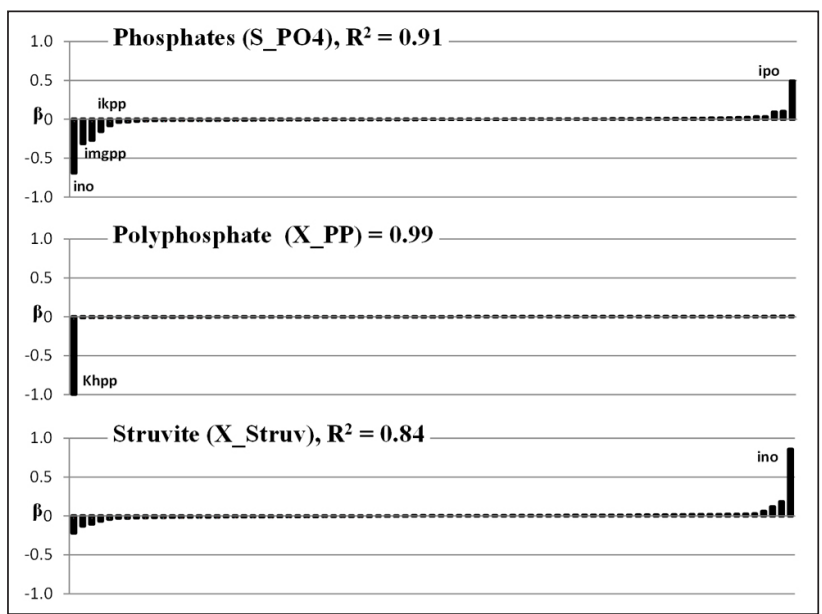

Figure 15. SRC results for sensitivity analysis of phosphates (S_PO4), Poly P (X_PAO_PP) and struvite precipitation (X_Struv) to UCTSDM3P parameters for EBPR WAS digestion

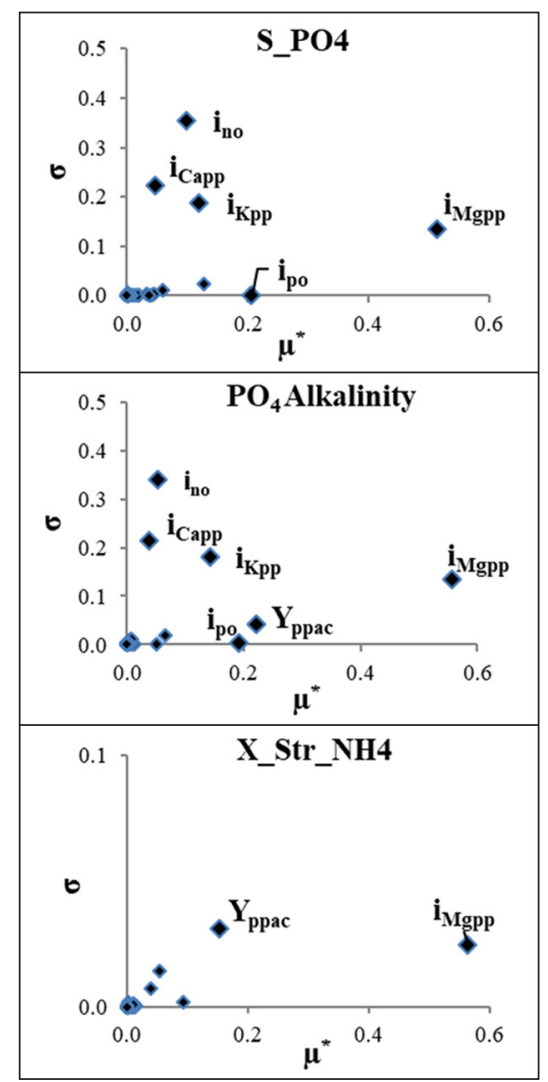

Figure 16. Morris screening results for sensitivity analysis of phosphates (S_PO4), Poly P (X_PAO_PP) and struvite precipitation (X_Struv) to UCTSDM3P parameters for EBPR WAS digestion 
For AD systems that treat WAS from BEPR plants, containing PAOs and PP, significant alkalinity gets generated from OP (through $\mathrm{MePO}_{3}+\mathrm{H}_{2} \mathrm{O} \rightarrow \mathrm{Me}^{+}+\mathrm{H}_{2} \mathrm{PO}_{4}^{-}$). The $\mathrm{H}_{2} \mathrm{PO}_{4}^{-}$is a nonreference species for the $\mathrm{OP}$ weak acid system that results from the breakdown of PP in the AD. It is noted further that since the phosphate weak acid/base sub-system (for $\mathrm{H}_{2} \mathrm{PO}_{4} / \mathrm{HPO}_{4}{ }^{2}$ speciation) has a $\mathrm{pK}_{\mathrm{p} 2}$ value at 7.13 , some of the $\mathrm{H}_{2} \mathrm{PO}_{4}{ }^{-}$consumes $\mathrm{H}_{2} \mathrm{CO}_{3}{ }^{*}$ Alk.

This substantiates the observations in Fig. 14, where the $\mathrm{pH}$ is also sensitive to changes in $Y_{\mathrm{ppac}}$. It was noted that the $i_{\mathrm{mgpp}}$ parameter controls the $\mathrm{Mg}$ content of $\mathrm{PP}$ and hence the amount of $\mathrm{Mg}$ eventually available (after PP hydrolysis) to promote $\mathrm{P}$ precipitation. Therefore, increasing this $\mathrm{Mg}$ content in PP would increase the $\mathrm{P}$ precipitation as struvite causing a decreased $\mathrm{OP}$ concentration in the aqueous phase. Moreover, the utilisation of $\mathrm{OP}$ with $\mathrm{P}$ precipitation brings about further adjustments in establishment of the system's alkalinity and $\mathrm{pH}$.

\section{Metals (Mg, $\mathrm{K}$ and $\mathrm{Ca}$ )}

The three main metallic ions released with PP breakdown in AD are $\mathrm{Mg}, \mathrm{K}$ and $\mathrm{Ca}$. The $\mathrm{Mg}$ and $\mathrm{K}$ are most influenced by changes in their respective molar contents in PP (i.e., $i_{\text {mgpp }}$ for $\mathrm{Mg}$ and $i_{\text {kpp }}$ for K) (see Fig. 17). The molar fraction of $\mathrm{Ca}$ in PP is usually significantly smaller than that for $\mathrm{Mg}$ and $\mathrm{K}$ - hence $i_{\text {capp }}$ doesn't seem to have a significant impact on $\mathrm{Ca}$ concentration in the aqueous phase. Instead, the parameters likely to influence system $\mathrm{pH}$, hence $\mathrm{Ca}$ precipitation potential (to calcium phosphate or calcium carbonate), are indicated to have an impact. These parameters include $i_{\text {no }}, i_{\text {ho }}, i_{\text {oo }}$ and $K_{\text {Cap }}$ (the rate constant for ACP precipitation). The Morris screening results also show that $i_{\text {kpp }}$ and $i_{\text {mgpp }}$ are significant parameters for $\mathrm{K}$ and $\mathrm{Mg}$, respectfully (see Fig. 18). However, $Y_{\text {ppac }}$ is included as the most significant of parameters for $\mathrm{Ca}$ and $\mathrm{Mg}$. It is expected then that $Y_{\mathrm{ppac}}$ would also be important for $\mathrm{K}$, but this is not reflected in the results. The probable reason for this is due to $\mathrm{K}$ being the only metal that was released and usually least likely to participate in mineral precipitation ( $\mathrm{K}$ - struvite, $\mathrm{MgKPO}_{4}$, has a high solubility product relative to the ionic product of $\mathrm{Mg}, \mathrm{K}$ and $\mathrm{PO}_{4}{ }^{3-}$ in the aqueous phase). This may amplify the influence of $i_{\text {kpp }}$ relative to other parameters.

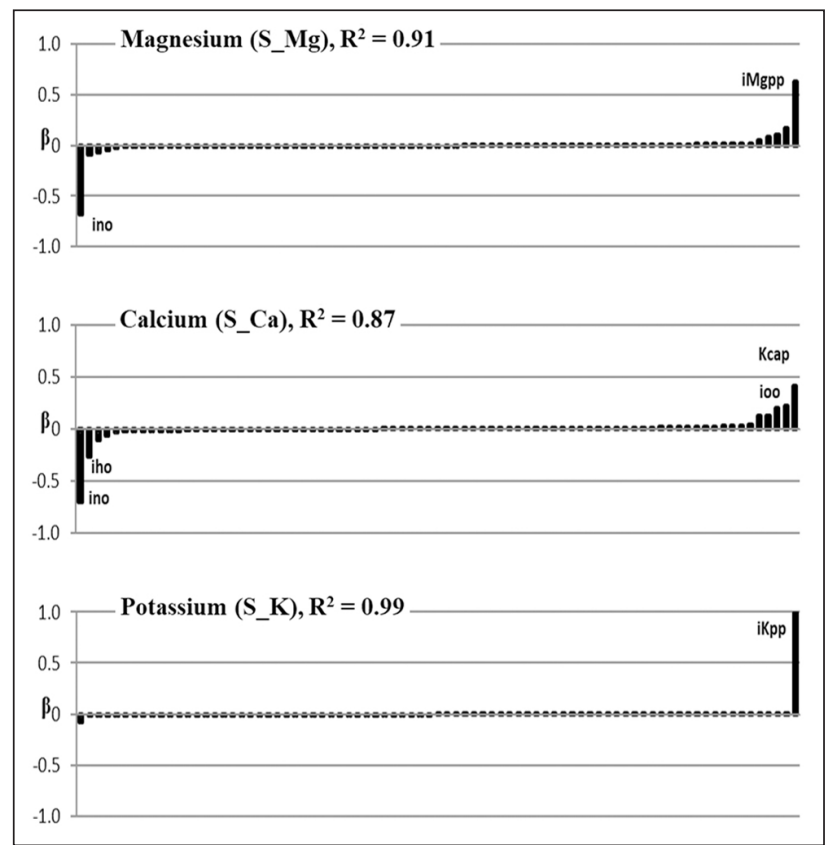

Figure 17. SRC results for sensitivity analysis of magnesium (S_Mg), calcium (S_Ca) and potassium (S_K) to UCTSDM3P parameters for EBPR WAS digestion

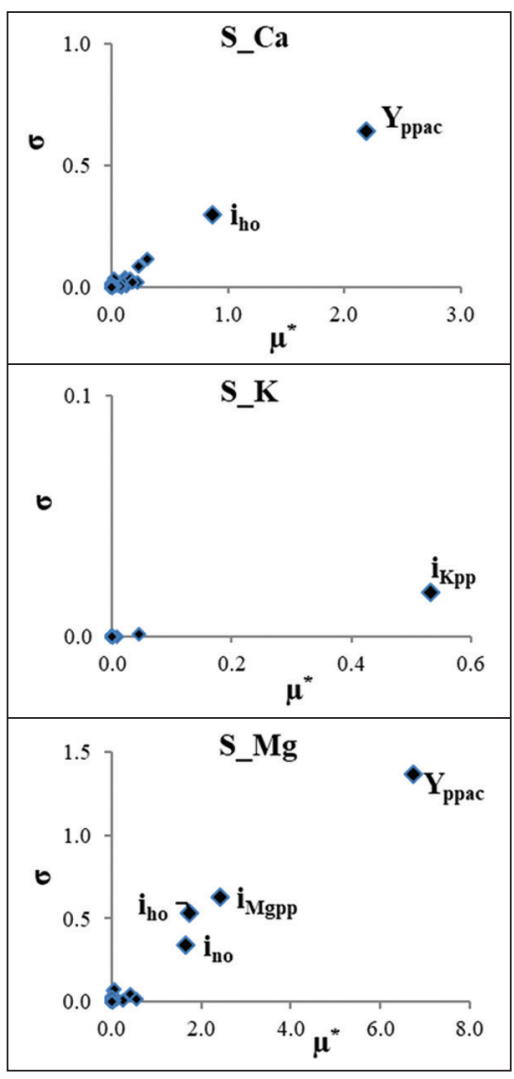

Figure 18. Morris screening results for sensitivity analysis of magnesium (S_Mg), calcium (S_Ca) and potassium (S_K) to UCTSDM3P parameters for EBPR WAS digestion

\section{CONCLUSION}

A sensitivity analysis was performed on ASM2-3P and UCTSDM3P (which together form PWM_SA) models using the standardized regression coefficient (SRC) and the Morris screening methods. The sensitivity analysis was useful towards detection of the significant parameters (prioritisation, using the SRC method) and non-influential parameters (with low $\mu$ and low $\sigma$, hence can be 'fixed', using the Morris screening method). For these sampled parameter sets, simulations have been conducted and predicted model outputs were compared with observed experimental outputs (Ikumi et al., 2015).

From this investigation it can be noted that various parameters, which are not normally measured at WRRFs, may require attention for future application of mathematical models in decision-making processes for WRRFs. These parameters include: for the AS system, $f_{\text {Sup }}, f_{\text {sbsa }}, m_{\text {uano }}$ and biodegradable organics elemental composition; and for the $\mathrm{AD}$ system fed WAS, the parameters driving kinetics of hydrolysis (i.e, $\mathrm{Ks}_{\text {bohyd }}$ and $\mathrm{kM}_{\text {bohyd }}$ ) and substrate elemental composition $\left(i_{\mathrm{no}}, i_{\mathrm{oo}}, i_{\mathrm{ho}}, i_{\mathrm{po}}, i_{\mathrm{mgpp}}, i_{\mathrm{kpp}}\right.$ and $\left.i_{\text {capp }}\right)$. The development of sophisticated augmented batch tests that work together with mathematical model parameter estimation techniques (i.e., as proposed by Botha and Ekama, 2015) could be used towards this process.

However, although some of the significant parameters could be applied generically for different systems (e.g., the yield values and endogenous death rates), other paramaters may require measurements specific to the system being designed or operated in order to obtain accurate predictions of system response.

\section{ACKNOWLEDGEMENTS}

This research was supported by the Water Research Commission, the National Research Foundation and the University of Cape Town and is published with their permission. 


\section{REFERENCES}

BATSTONE DJ, KELLER J, ANGELIDAKI I, KALYUZNHYI SV, PAVLOSTATHIS SG, ROZZI A, SANDERS WTM, SIEGRIST $\mathrm{H}$ and VAVILIN VA (2002) Anaerobic digestion model No. 1 (ADM1). Scientific and Technical Report No 9. International Water Association (IWA), London.

BATSTONE DJ, AMERLINCK Y, EKAMA G, GOEL R, GRAU P, JOHNSON B, KAYA I, STEYER J-P, TAIT S, TAKÁCS I, VANROLLEGHEM PA, BROUCKAERT CJ and VOLCKE E (2012) Towards a generalized physicochemical framework. Water Sci. Technol. 66 (6) 1147-1161. https://doi.org/10.2166/wst.2012.300

BROUCKAERT CJ, IKUMI DS and EKAMA GA (2010) A 3-phase anaerobic digestion model. Proc. $12^{\text {th }}$ IWA Anaerobic Digestion Conference (AD12), 1-4 November 2010, Guadalajara, Mexico.

BRUN R, KÜHNI M, SIEGRIST H, GUJER W and REICHERT P (2002) Practical identifiability of ASM2d parameters-systematic selection and tuning of parameter subsets. Water Res. 36 4113-4127. https:// doi.org/10.1016/S0043-1354(02)00104-5

CAMPOLONGO F, CARIBONI J and SALTELLI A (2007) An effective screening design for sensitivity analysis of large models. Environ. Model. Softw. 22 1509-1518. https://doi.org/10.1016/j.envsoft.2006.10. 004

COROMINAS LL, RIEGER L, TAKÁCS I, EKAMA GA, HAUDUC $\mathrm{H}$, VANROLLEGHEM PA, OEHMEN A, GERNAEY KV, VAN LOOSDRECHT MC and COMEAU Y (2010) New framework for standardized notation in wastewater treatment modelling Water Sci. Technol. 61 (4) 841-857. https://doi.org/10.2166/wst.2010.912

COSENZA A, MANNINA G, VANROLLEGHEM PA and NEUMANN MB (2013) Global sensitivity analysis in wastewater applications: A comprehensive comparison of different methods. Environ. Model. Softw. 49 40-52. https://doi.org/10.1016/j.envsoft.2013.07.009

EKAMA GA and WENTZEL MC (2004) A predictive model for the reactor inorganic suspended solids concentration in activated sludge systems. Water Res. 38 (19) 4093-4106. https://doi.org/10.1016/j. watres.2004.08.005

EKAMA GA, WENTZEL MC and SÖTEMANN SW (2006) Mass balanced-based plant-wide wastewater treatment plant models Part 2: Tracking the influent inorganic suspended solids. Water SA. 32 (3) 277-285. https://doi.org/10.4314/wsa.v32i3.5272

EKAMA GA (2009) Using bio-process stoichiometry to build a steady state plant-wide wastewater treatment plant model. Water Res. 43 (8) 2101-2120. https://doi.org/10.1016/j.watres.2009.01.036

FLORES-ALSINA $\mathrm{X}$, SOLON K, KAZADI MBAMBA C, TAIT S, JEPPSSON U, GERNAEY KV and BATSTONE DJ (2016) Modelling phosphorus, sulphur and iron interactions during the dynamic simulation of anaerobic digestion processes. Water Res. 95 370-382. https://doi.org/10.1016/j.watres.2016.03.012

GAMERITH V, NEUMANN MB and MUSCHALLA D (2011) Applied global sensitivity analysis in sewer flow and water quality modelling. Proc. $12^{\text {th }}$ International Conference of Urban Drainage, 10-15 September 2011, Porto Alegre, Brazil.

GHOOR T (2020) Developments in anaerobic digestion modelling. Department of Civil Engineering, University of Cape Town, Rondebosch, 7701, Western Cape, South Africa

HARDING TH, IKUMI DS and EKAMA GA (2010) A steady state stoichiometric model describing the anaerobic digestion of biological excess phosphorus removal waste activated sludge. Research Report W132 (MSc thesis), Department of Civil Engineering, University of Cape Town, South Africa.

HAUDUC H, RIEGER L, TAKÁCS I, HÉDUIT A, VANROLLEGHEM PA and GILLOT S (2010) A systematic approach for model verification: Application on seven published activated sludge models. Water Sci. Technol. 61 (4) 825-839. https://doi.org/10.2166/wst.2010.898

HENZE M, GUJER W, MINO T, MATSUO T, WENTZEL MC and MARAIS GVR (1995) Activated sludge model No.2 (ASM2). IWA Scientific and Technical Report No. 3. IWA Publishing, London.

HENZE M, VAN LOOSDRECHT MCM, EKAMA GA and BRDJANOVIC D (2008) Biological Wastewater Treatment: Principles, Design and Modelling. IWA Publishing, London. 620 pp.

HERMAN JD, KOLLAT JB, REED PM and WAGENER T (2013) Technical Note: Method of Morris effectively reduces the computational demands of global sensitivity analysis for distributed watershed models. Hydrol. Earth Syst. Sci. 10 (4) 4275-4299. https:// doi.org/10.5194/hessd-10-4275-2013
HILL DT and BARTH CL (1977) A dynamic model for simulation of animal waste digestion. J. Water Pollut. Control Fed. 49 (10) 21292143.

IKUMI DS (2011) The development of a three-phase plant-wide mathematical model for sewage treatment. $\mathrm{PhD}$ thesis, Water Research Group (WRG), Department of Civil Engineering. University of Cape Town.

IKUMI D, VANROLLEGHEM PA, BOUCKAERT V, NEUMANN $M$ and EKAMA G (2014) Towards calibration of phosphorus (P) removal plant-wide models. Proc. $4^{\text {th }}$ IWA/WEF Wastewater Treatment Modelling Seminar (WWTmod2014), 30 March-2 April 2014, Spa, Belgium. 197-206.

IKUMI DS, HARDING TH, VOGTS M, LAKAY MT, MAFUNGWA HZ, BROUCKAERT CJ and EKAMA GA (2015) Mass balances modelling over wastewater treatment plants III. WRC Report No. 1822/1/14. Water Research Commission, Pretoria.

IKUMI DS, HARDING TH and EKAMA GA (2014) Plant-wide wastewater treatment modelling (1) - Biodegradability of wastewater and activated sludge organics in anaerobic digestion. Water Res. 56 (1) 267-279. https://doi.org/10.1016/j.watres.2014.02.008

IKUMI DS, HARDING TH, VOGTS M, LAKAY MT, MAFUNGWA HZ, BROUCKAERT CJ and EKAMA GA (2015) Mass balances modelling over wastewater treatment plants III. WRC Report No. 1822/1/14. Water Research Commission, Pretoria.

KAZADI MBAMABA C, FLORES-ALSINA X, BATSTONE DJ and TAIT S (2015) A systematic study of multiple minerals precipitation modelling in wastewater treatment. Water Res. 85 359-370. https:// doi.org/10.1016/j.watres.2015.08.041

LIZARRALDE I, FERNÁNDEZ-ARÉVALOA T, BROUCKAERT CJ, VANROLLEGHEM PA, IKUMI DS, EKAMA GA, AYESA E and GRAU P (2015) A new general methodology for incorporating physicochemical transformations into multi-phase wastewater treatment process models. Water Res. 74 (5) 239 -256. https://doi. org/10.1016/j.watres.2015.01.031

LOEWENTHAL RE, KORNMULLER URC and VAN HEERDEN EP (1994) Modelling struvite precipitation in anaerobic treatment systems. Water Sci. Technol. 30 (12) 107-116. https://doi.org/10.2166/ wst.1994.0592

MANNINA G, COSENZA A, VANROLLEGHEM PA and VIVIANI G (2011) A practical protocol for calibration of nutrient removal wastewater treatment models. J. Hydroinf. 13 (4) 575-595. https:// doi.org/10.2166/hydro.2011.041

McCARTY PL (1975) Stoichiometry of biological reactions. Prog. Water Technol. 7 (1) 157-172.

MOOSBRUGGER RE, WENTZEL MC, EKAMA GA and MARAIS GVR (1993b) A five-pH point titration method for determining the carbonate and SCFA weak acid/bases in anaerobic systems. Water Sci. Technol. 28 (2) 237-246. https://doi.org/10.2166/wst.1993.0112

MORRIS MD (1991) Factorial sampling plans for preliminary computational experiments. Technometrics. 33 (2) 161-174. https:// doi.org/10.1177/001872086700900503

MUSVOTO EV, WENTZEL MC and EKAMA GA (2000) Integrated chemical-physical processes modelling I. Development of a kinetic based model for weak acid/base systems. Water Res. 34 (6) 18571867. https://doi.org/10.1016/S0043-1354(99)00334-6

NEUMANN MB (2012) Comparison of sensitivity analysis methods for pollutant degradation modelling: A case study from drinking water treatment. Sci. Total Environ. 433 530-537. https://doi.org/10.1016/j. scitotenv.2012.06.026

NOYKOVA NA and GYLLENBERG M (2000) Sensitivity analysis and parameter estimation in a model of anaerobic wastewater treatment processes with substrate inhibition. Bioprocess Biosyst. Eng. 23 (4) 343-349. DOI: https://doi.org/10.1007/s004499900169

SALTELLI A, TARANTOLA S and CHAN KP (1999) A quantitative model-independent method for global sensitivity analysis of model output. Technometrics. 41 (1) 39-56. https://doi.org/10.1080/004017 06.1999.10485594

SALTELLI A, TARANTOLA S, CAMPOLONGO F and RATTO M (2004) Sensitivity Analysis in Practice, a Guide to Assessing Scientific Models. Wiley, New York.

SILVA MI and DE BORTOLI AL (2020) Sensitivity analysis for verification of an anaerobic digestion model. Int. J. Appl. Comput. Math. 6 (38). DOI: https://doi.org/10.1007/s40819-020-0791-z 
SOBOL' I (1993) Sensitivity analysis for non-linear mathematical models. Math. Model. Comput. Exp. (Engl. Transl.). 1 407-414.

SOLON K, FLORES-ALSINA X, KAZADI MBAMBA C, IKUMI D, VOLCKE EIP, VANEECKHAUTE C, EKAMA GA, VANROLLEGHEM PA, BATSTONE DJ, GERNAEY KV and JEPPSSON U (2017) Plant-wide modelling of phosphorus transformations in wastewater treatment systems: Impacts of control and operational strategies. Water Res. 113 97-110. https://doi org/10.1016/j.watres.2017.02.007

SÖTEMANN SW, RISTOW NE, WENTZEL MC and EKAMA GA (2005) A steady-state model for anaerobic digestion of sewage sludges. Water SA. 31 (4) 511-527. https://doi.org/10.4314/wsa.v31i4 5143

SÖTEMANN SW, VAN RENSBURG P, RISTOW NE, WENTZEL MC, LOEWENTHAL RE and EKAMA GA (2005b) Integrated chemical, physical and biological processes modelling Part 2 - Anaerobic digestion of sewage sludges Water SA. 31 545-568. https://doi.org/ 10.4314/wsa.v31i4.5145

TAIT S, SOLON K, VOLCKE EIP and BATSTONE DJ (2012) A unified approach to modelling wastewater chemistry: model corrections. Proc. $3^{\text {rd }}$ Wastewater Treatment Modelling Seminar (WWTmod2012), 26-28 February 2012, Mont-Sainte-Anne, Quebec, Canada.

VANHOOREN $\mathrm{H}$, MEIRLAEN J, AMERLINCK Y, CLAEYS F, VANGHELUWE $\mathrm{H}$ and VANROLLEGHEM PA (2003) WEST: modelling biological wastewater treatment. J. Hydroinf. 5 27-50. https://doi.org/10.2166/hydro.2003.0003

VAN RENSBURG P, MUSVOTO EV, WENTZEL MC and EKAMA GA (2003) Modelling multiple mineral precipitation in anaerobic digester liquor. Water Res. 37 (13) 3087-3097. https://doi.org/10.1016/ S0043-1354(03)00173-8
VANROLLEGHEM PA, INSEL G, PETERSEN B, SIN G, DE PAUW D, NOPENS I, WEIJERS S and GERNAEY K (2003) A comprehensive model calibration procedure for activated sludge models. Proc. WEF $26^{\text {th }}$ Annual Technical Exhibition and Conference (WEFTEC, 2003), 11-15 October 2003, Los Angeles. https://doi.org/10.2175/1938 64703784639615

VOLCKE EIP, VAN LOOSDRECHT MCM and VANROLLEGHEM PA (2006) Continuity-based model interfacing for plant-wide simulation: A general approach. Water Res. 40 2817-2828. https:// doi.org/10.1016/j.watres.2006.05.011

WANG R, YONGMEI L, CHEN W, ZOU J and CHEN Y (2016) Phosphate release involving PAOs activity during anaerobic fermentation of EBPR sludge and the extension of ADM1. Chem. Eng. J. 297 (1) $436-$ 447. https://doi.org/10.1016/j.cej.2015.10.110

WENTZEL MC (1988) Biological excess phosphorus removal in activated sludge systems. PhD thesis, Water Research Group, Department of Civil Engineering, University of Cape Town.

WENTZEL MC, EKAMA GA, DOLD PL and MARAIS GVR (1990) Biological excess phosphorous removal - steady state process design Water SA. 16 (1) 29-48.

ZHAO X, LI L, WU D, XIAO T, MA Y and PENG X (2019) Modified Anaerobic Digestion Model No. 1 for modelling methane production from food waste in batch and semi-continuous anaerobic digestions. Bioresour. Technol. 271 109-117. https://doi.org/10.1016/j.biortech. 2018.09.091 